\title{
ELECTRON-ION RECOMBINATION RATE COEFFICIENTS AND PHOTOIONIZATION CROSS SECTIONS FOR ASTROPHYSICALLY ABUNDANT ELEMENTS. VIII. Ar XIII WITH NEW FEATURES
}

\author{
Sultana N. Nahar \\ Department of Astronomy, The Ohio State University, Columbus, OH 43210; nahar@astronomy.ohio-state.edu \\ Received 2004 June 28; accepted 2004 September 15
}

\begin{abstract}
Ar XIII is found to be unique with new features in electron-ion recombination not seen in any other ion. The ion has been studied with the unified method, which provides a theoretically self-consistent set of atomic parameters for the inverse processes of photoionization and total electron-ion recombination. Unified method subsumes both the radiative recombination (RR) and dielectronic recombination (DR) within the framework of close-coupling formulations using the $\boldsymbol{R}$-matrix method. A set of four DR "bumps," two in the low-temperature and two in the high-temperature regions, is found to exist in the recombination rates of $\mathrm{Ar} \mathrm{XIV}+e \rightarrow \mathrm{Ar}$ XIII. This is in contrast to two typical DR "bumps," one at high temperature common for most ions and one at low temperature depending on the presence of near threshold autoionizing resonances in the bound-free process. Large-scale ab initio calculations have been carried out for photoionization and electron-ion recombination cross sections of $\mathrm{Ar}$ XIII. The ion is represented by a large close-coupling eigenfunction expansion of 37 core $\mathrm{Ar}$ XIV states from $n=2$ and 3 complexes. This enables core excitations of type $\Delta n=0$ and 1 . The $\Delta n=1$ transitions have much higher radiative decay rates than those of $\Delta n=0$ and cause the fourth DR bump around $2 \times 10^{6} \mathrm{~K}$. For a large number of bound states, Ar XIII exhibits more extensive resonant structures and wider PEC (photoexitation-of-core) resonances for $n=3$ core states than those of $n=2$ states. Hence, the high-energy regions of photoionization and recombination are dominated by these structures. A total of 684 bound states with valence electron $n \leq 10$ and $l \leq 9$ are found for Ar XIII. Total and partial photoionization cross sections of all bound states, state-specific recombination rates of 561 bound states, and total recombination rate coefficients at a large temperature range are presented for Ar XIII.
\end{abstract}

Subject headings: atomic data - atomic processes

\section{INTRODUCTION}

Ar XIII has been studied less compared to many other ions and relatively little reliable atomic data are available for this ion. However, reliable atomic parameters are important to interpret the large number of high-resolution spectra being taken by observatories such as HST, EUVE, and FUSE, over optical to extreme ultraviolet wavelength ranges and to understand the physical conditions and properties of astrophysical and laboratory plasmas. Photoionization of Ar XIII was studied under the Opacity Project (OP) (Seaton et al. 1995-1996) using a relatively smaller wavefunction expansion (Nahar \& Pradhan 1992a). Oscillator strengths for allowed transitions including relativistic effects in the Breit-Pauli $\boldsymbol{R}$-matrix approximation were obtained by Nahar (2000). Earlier works on electron-ion recombination include radiative recombination (RR) rates by Aldrovandi \& Pequignot (1974) and dielectronic recombination (DR) rates by Shull \& van Steenberg (1982), Gu (2003), and Altun et al. (2004).

The present work reports results of a self-consistent study of the two inverse processes photoionization and electron-ion recombination of $\mathrm{Ar}$ XIII $+h \nu \leftrightarrow \mathrm{Ar} \mathrm{XIV}+e$ employing the unified method for the total recombination. The method, in principle, provides the most accurate total recombination $(R R+D R)$ rate coefficients with considerations of interference and channel coupling effects. Total and state-specific recombination rate coefficients of other carbon-like ions, e.g., from $\mathrm{C}$ I to $\mathrm{S}$ XI, were presented earlier (Nahar 1995, 1996a). However, Ar XIII appears to be a unique ion with differences to these ions. Unlike to their cores, the $n=3$ core states of Ar XIII are separated by a large energy gap from those of $n=2$. A smaller wavefunction expansion of eight terms, compared to the present 37 terms, was used in the earlier close-coupling $\boldsymbol{R}$-matrix calculations. Inclu- sion of highly excited core states in the present wavefunction expansion has revealed new features for photoionization and recombination for Ar XIII that are reported herein.

The present series of reports (see Nahar \& Pradhan 1997, hereafter Paper I) aims at studying and presenting accurate atomic parameters for photoionization and total $(e+$ ion $)$ recombination for astrophysical models on a variety of applications, such as planetary nebulae, active galactic nuclei, and stars. The ab initio unified treatment of Nahar \& Pradhan (1992b, 1994, 1995, 2004) provides (1) total recombination rates that is valid for all temperatures, (2) level-specific recombination rates for a large number of atomic levels, and (3) self-consistent sets of photoionization cross sections, $\sigma_{\mathrm{PI}}$, and electron-ion recombination rate coefficients, $\alpha_{R}$, which reduce the uncertainty in astrophysical models. In contrast, the other methods, mainly the isolated resonance approximation with distorted wave (e.g., Gu 2003; Altun et al. 2004) considers limited number of resonances, although convergence with respect to the principle quantum number could be checked for optimum contribution and can obtain only the DR rates. The RR rates, which do not include any resonances, may be calculated using a distorted wave or central field or hydrogenic approximation separately to add to DR for the total.

\section{THEORY}

The unified treatment for the total electron-ion recombination, which subsumes both the radiative and dielectronic recombination processes in an ab initio manner, enables self-consistent results for $\sigma_{\mathrm{PI}}$ and $\alpha_{R}$ by using the same wavefunction that describes the $(e+i o n)$ system for both processes, photoionization and recombination. The details of the theory are given in earlier 
papers (Nahar \& Pradhan 1994, 1995; Nahar 1996b). A brief outline of the method is given below.

\subsection{The Coupled Channel Wavefunction Expansion}

In the close-coupling (CC) approximation (Seaton 1987), the "target" or the "core" ion is represented by an $N$-electron system, while the interacting $(N+1)$ th electron is bound or in the continuum depending on its energy. The total wavefunction, $\Psi_{E}$, of the $(N+1)$ electron system for any symmetry $S L \pi$ is represented by an expansion of the eigenfunctions of the target ion, $\chi_{i}$, coupled to the $(N+1)$ th electron function, $\theta_{i}$, as

$$
\Psi_{E}(e+\text { ion })=A \sum_{i} \chi_{i}(\text { ion }) \theta_{i}+\sum_{j} c_{j} \Phi_{j},
$$

where the target is in a specific state $S_{i} L_{i} \pi_{i}$ and the $(N+1)$ th electron is in a channel labeled $S_{i} L_{i} \pi_{i} k_{i}^{2} \ell_{i}(S L \pi)$, where $k_{i}^{2}$ is its kinetic energy. The $\Phi_{j}$-values are bound channel functions of the $(N+1)$-electron system that account for short range correlation and the orthogonality between the continuum and the bound electron orbitals.

Substitution of the wavefunction expansion in

$$
H_{N+1} \Psi_{E}=E \Psi_{E},
$$

where the $(N+1)$-electron Hamiltonian is

$$
H_{N+1}=\sum_{i=1}^{N+1}\left\{-\nabla_{i}^{2} 2-\frac{2 Z}{r_{i}}+\sum_{j>i}^{N+1} \frac{2}{r_{i j}}\right\},
$$

introduces a set of coupled equations that are solved using the $\boldsymbol{R}$-matrix approach (Burke et al. 1971; Seaton et al. 1995-1996). The continuum wavefunction, $\Psi_{F}$, describes the scattering process with the free electron interacting with the target at positive energies $(E>0)$, while at negative total energies $(E \leq 0)$, the solutions correspond to pure bound states $\Psi_{B}$. The complex resonant structures in photoionization and recombination result from couplings between continuum channels that are open $\left(k_{i}^{2}>0\right)$ and bound channels that are closed $\left(k_{i}^{2}<0\right)$ and form at electron energies $k_{i}^{2}$ corresponding to the Rydberg series of states converging on to the target thresholds.

\subsection{Photoionization}

The transition matrix elements for photoionization and recombination can be obtained using the bound and continuum wavefunctions as

$$
\left\langle\Psi_{B}|| \boldsymbol{D} \| \Psi_{F}\right\rangle,
$$

where $\boldsymbol{D}$ is the dipole operator. In "length" form, $\boldsymbol{D}_{L}=\sum_{i} r_{i}$, and in "velocity" form, $\boldsymbol{D}_{V}=-2 \sum_{i} \Delta_{i}$, where the sum corresponds to number of electrons. The transition matrix element with the dipole operator can be reduced to the generalized line strength defined, in either length form as

$$
S_{L}=\left|\left\langle\Psi_{j}|| \boldsymbol{D}_{L}|| \Psi_{i}\right\rangle\right|^{2}=\left|\left\langle\Psi_{f}\left|\sum_{j=1}^{N+1} r_{j}\right| \Psi_{i}\right\rangle\right|^{2},
$$

or in velocity form as

$$
S_{V}=E_{i j}^{-2}\left|\left\langle\Psi_{j}|| \boldsymbol{D}_{V}|| \Psi_{i}\right\rangle\right|^{2}=\omega^{-2}\left|\left\langle\Psi_{f}\left|\sum_{j=1}^{N+1} \frac{\partial}{\partial r_{j}}\right| \Psi_{i}\right\rangle\right|^{2} .
$$

where $\omega$ is the incident photon energy in Rydberg units, and $\Psi_{i}$ and $\Psi_{f}$ are the initial and final state wave functions. The photoionization cross section $\left(\sigma_{\mathrm{PI}}\right)$ is proportional to the generalized line strength $(S)$,

$$
\sigma_{\mathrm{PI}}=\frac{4 \pi^{2}}{3 c} \frac{1}{g_{i}} \omega \boldsymbol{S},
$$

where $g_{i}$ is the statistical weight factor of the bound state.

\subsection{Unified $(e+$ ion) Recombination}

The total and state-specific recombination rate coefficients are obtained using the unified treatment within the close-coupling approximation using the same wavefunction as for photoionization. The infinite number of recombined states of the electronion system are divided into two groups: (1) low- $n$ states, $n \leq n_{0}$, and (B) high- $n$ states, $n_{0}<n \leq \infty$, with $n_{0} \sim 10$.

The recombination cross sections, $\sigma_{\mathrm{RC}}(i)$, and the recombination rate coefficients, $\alpha_{R}(i ; T)$ of the low- $n$ bound states of group A are obtained from the detailed partial photoionization cross sections, $\sigma_{\mathrm{PI}}(i \rightarrow j)$, through the Milne relation (principle of detailed balance):

$$
\sigma_{\mathrm{RC}}(j)=\sigma_{\mathrm{PI}}(i) \frac{g_{i}}{g_{j}} \frac{h^{2} \omega^{2}}{4 \pi^{2} m^{2} c^{2} v^{2}},
$$

where $g_{i}$ and $g_{j}$ are the statistical weight factors of the recombining and recombined states, respectively, and $v$ is the photoelectron velocity. The recombining ion is assumed to be in the ground state.

Recombination rate coefficients of individual states are then obtained by averaging the recombination cross sections over the Maxwellian electron distribution, $f(v)$, at a given temperature as

$$
\alpha_{R}(i ; T)=\int_{0}^{\infty} v f(v) \sigma_{R C}(i, v) d v .
$$

The sum of these individual rates $\sum_{i} \alpha_{R}(i, T)$ provides the contribution of the low- $n$ bound states to the total recombination rate. As the cross sections include the detailed structures of autoionizing resonances, the sum corresponds to the inclusion of RR and DR in an unified and ab initio manner.

Recombination into the group B states $\left(n_{0}<n \leq \infty\right)$ is dominated by DR via the high- $n$ resonances converging on to the thresholds in the core ion, and the background recombination via RR is negligibly small. The theory of DR developed by Bell \& Seaton (1985) is extended (Nahar \& Pradhan 1994; Nahar 1996b) to compute the DR collision strengths, $\Omega(D R)$, in this energy region. Contributions come from the DR resonances belonging to excited core states that decay to the ground state via dipole allowed transitions. The recombination cross section is obtained as

$$
\sigma_{\mathrm{RC}}(\mathrm{DR})=\frac{\pi}{g_{i} k^{2}} \Omega(\mathrm{DR}) a_{0}^{2},
$$

where $k^{2}$ is the energy of the free electron. The value of $\Omega(\mathrm{DR})$ is summed over all contributing symmetries $S L \pi$. Both the detailed and resonance-averaged forms of DR collision strength are obtained for resolution tests. The DR rate coefficients are obtained by averaging over the Maxwellian distribution function.

For consistency check on the DR calculations, independent $\boldsymbol{R}$-matrix close-coupling scattering calculation is carried out for electron impact excitation (EIE) collision strengths, $\Omega$ (EIE), at 
excited target thresholds using the same $\mathrm{CC}$ wavefunction expansion used for photoionization and recombination. As the energy from below the threshold reaches the threshold, the $\Omega(D R)$ should merge to $\Omega$ (EIE) as the captured electron via DR is released by excitation of the core.

The RR-type "background" contributions from the high- $n$ states, $n_{0}<n \leq \infty$, to the total recombination rate at all temperatures are included in the hydrogenic approximation (Nahar 1996b). These contributions are usually negligible except at very low temperatures where electron energies are not high enough for the core excitations. The low-energy recombination to the infinite number of high- $n$ states typically shows a characteristic rapid rise in $\alpha_{R}$ in the low- $T$ region.

\section{COMPUTATIONS}

The present wavefunction expansion for Ar XIII consists of the 37 lowest target terms of the $n=2$ and 3 complexes (listed in Table 1). The first eight terms are from configurations $2 s^{2} 2 p$, $2 s 2 p^{2}$, and $2 p^{3}$ of the $n=2$ complex, as used in the earlier calculations by Nahar \& Pradhan (1992b), and the additional 29 terms are from the $n=3$ complex. A large energy gap exists between terms of the $n=2$ and $n=3$ complexes, the last $n=2$ term being at 8 ryd, while the first $n=3$ term is at over 31 ryd. Such an energy gap is often the criterion for the cutoff limit for wavefunction expansion as no new bound states are formed from these highly excited core states and the correlations due to them are usually much weaker.

The target, Ar XIV, wavefunctions are obtained from atomic structure calculations using the code SUPERSTRUCTURE (Eissner et al. 1974), which employs the scaled Thomas-FermiDirac potential to generate the one-electron orbitals. The set of spectroscopic and correlation configurations, and the values of the Thomas-Fermi scaling parameter $\left(\lambda_{\mathrm{nl}}\right)$ for each orbital, are given in Table 1. The table presents term energies of target Ar XIV used in the calculations. The calculated target energies have been replaced by the observed energies whenever available (term energies without the asterisks in the table). This improves the accuracy of resonance positions in the photoionization cross sections. The observed energies in Table 1 are from National Institute of Standards and Technology (NIST) database and were measured by R. L. Kelly (unpublished, data available at NIST).

The second sum in the wavefunction expansion, equation (1), includes all possible $(N+1)$-electron configurations with maximum occupancies up to $2 p^{4}, 3 s^{2}, 3 p^{2}$, and $3 d^{2}$ for Ar XIII. All $S L \pi$ symmetries of the ( $e+$ ion) system formed from target states of the ion coupled with the interacting electron with partial waves $l \leq 9$ are included.

The $\boldsymbol{R}$-matrix calculations were carried out using the extended version of codes developed under the Iron Project (Hummer et al. 1993; Berrington et al. 1987, 1995; Nahar \& Pradhan 1995). Calculations were carried out for two or a few symmetries at a time because of computational problems with large dimensions, memory size requirements, and many CPU hours.

Spectroscopic identification of the large number of bound states was considerably challenging. The present version of the IP code for bound states, STGB, does not analyze the energy eigenvalues of the Hamiltonian for any spectroscopic identification. A computer program, PLSRAD, was written to analyze the bound states with the highest contributing channels, quantum defect numbers, to identify the Rydberg series of states and assign the spectroscopic notation. However, for closely lying states, identification may have uncertainties because of a similar amount of quantum defects.
TABLE 1

Target Terms in the Eigenfunction Expansions of Ar XiII

\begin{tabular}{|c|c|c|}
\hline Number & Term & $\begin{array}{c}E \\
\text { (ryd) }\end{array}$ \\
\hline \multicolumn{3}{|c|}{$n=2$ States } \\
\hline & $2 s^{2} 2 p\left({ }^{2} P^{o}\right)$ & 0.0 \\
\hline & $2 s 2 p^{2}\left({ }^{4} P^{e}\right)$ & 1.910198 \\
\hline & $2 s 2 p^{2}\left({ }^{2} D^{e}\right)$ & 3.743752 \\
\hline 4 & $2 s 2 p^{2}\left({ }^{2} S^{e}\right)$ & 4.687832 \\
\hline $5 \ldots \ldots \ldots$ & $2 s 2 p^{2}\left({ }^{2} P^{e}\right)$ & 5.025790 \\
\hline & $2 p^{3}\left({ }^{4} S^{o}\right)$ & 6.245461 \\
\hline $7 \ldots \ldots \ldots$ & $2 p^{3}\left({ }^{2} D^{o}\right)$ & 7.126292 \\
\hline 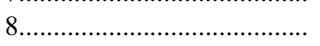 & $2 p^{3}\left({ }^{2} P^{o}\right)$ & 8.057424 \\
\hline \multicolumn{3}{|c|}{$n=3$ States } \\
\hline & $2 s^{2} 3 s\left({ }^{2} S\right)$ & 31.56629 \\
\hline $10 \ldots \ldots$ & $2 s^{2} 3 p\left({ }^{2} P^{o}\right)$ & 32.61456 \\
\hline 11 & $2 s 2 p 3 s\left({ }^{4} P^{o}\right)^{*}$ & 33.00461 \\
\hline 12 & $2 s^{2} 3 d\left({ }^{2} D\right)$ & 33.182154 \\
\hline $13 \ldots \ldots$ & $2 s 2 p 3 s\left({ }^{2} P^{o}\right)^{*}$ & 33.53359 \\
\hline $14 \ldots \ldots \ldots . . . .$. & $2 s 2 p 3 p\left({ }^{4} D\right)^{*}$ & 33.9535 \\
\hline 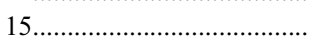 & $2 s 2 p 3 p\left({ }^{4} S\right)^{*}$ & 34.12207 \\
\hline 16 & $2 s 2 p 3 p\left({ }^{4} P\right)^{*}$ & 34.28115 \\
\hline 17 & $2 s 2 p 3 p\left({ }^{2} D\right)^{*}$ & 34.44365 \\
\hline 18 & $2 s 2 p 3 p\left({ }^{2} S\right)^{*}$ & 34.75660 \\
\hline 19 & $2 s 2 p 3 d\left({ }^{4} F^{o}\right)^{*}$ & 34.77136 \\
\hline 20 & $2 s 2 p 3 d\left({ }^{4} D^{o}\right)^{*}$ & 34.98291 \\
\hline 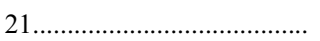 & $2 s 2 p 3 s\left({ }^{2} P^{o}\right)^{*}$ & 35.06681 \\
\hline 22 & $2 s 2 p 3 d\left({ }^{4} P^{o}\right)^{*}$ & 35.07163 \\
\hline 23 & $2 s 2 p 3 d\left({ }^{2} D^{o}\right)$ & 35.45923 \\
\hline 24 & $2 s 2 p 3 d\left({ }^{2} P^{o}\right)^{*}$ & 35.63076 \\
\hline $25 \ldots$ & $2 s 2 p 3 p\left({ }^{2} P\right)$ & 35.84257 \\
\hline 26 & $2 s 2 p 3 d\left({ }^{2} F^{o}\right)$ & 35.97162 \\
\hline 27 & $2 s 2 p 3 p\left({ }^{2} P\right)^{*}$ & 36.03974 \\
\hline 28 & $2 s 2 p 3 p\left({ }^{2} D\right)^{*}$ & 36.07177 \\
\hline $29 \ldots \ldots \ldots$ & $2 s 2 p 3 p\left({ }^{2} S\right)^{*}$ & 36.33810 \\
\hline 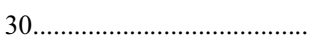 & $2 p^{2} 3 s\left({ }^{4} P\right)^{*}$ & 36.53905 \\
\hline 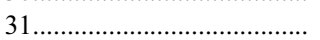 & $2 p^{2} 3 s\left({ }^{2} P\right)^{*}$ & 37.13357 \\
\hline 32 & $2 p^{2} 3 p\left({ }^{2} S^{o}\right)^{*}$ & 37.18915 \\
\hline 33 & $2 s 2 p 3 d\left({ }^{2} F^{o}\right)$ & 37.22526 \\
\hline 34 & $2 s 2 p 3 d\left({ }^{2} P^{o}\right)^{*}$ & 37.22654 \\
\hline 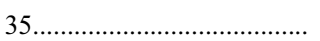 & $2 p^{2} 3 p\left({ }^{4} D^{o}\right)^{*}$ & 37.37029 \\
\hline 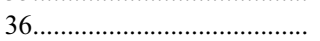 & $2 p^{2} 3 s\left({ }^{2} D\right)^{*}$ & 37.38289 \\
\hline 37 & $2 s 2 p 3 d\left(^{2} D^{o}\right)$ & 38.28871 \\
\hline
\end{tabular}

Notes.-Asterisk $(*)$ indicates that the term has not been observed. The observed energies are from NIST database measured by R. L. Kelly. The $n=2$ terms are separated from $n=3$ terms by a large energy gap. Atomic structure calculations for the target included the spectroscopic configurations are $2 s^{2} 2 p, 2 s 2 p^{2}, 2 p^{3}$, $2 s^{2} 3 s, 2 s^{2} 3 p, 2 s^{2} 3 d, 2 s 2 p 3 s, 2 s 2 p 3 p, 2 s 2 p 3 d, 2 p^{2} 3 s, 2 p^{2} 3 p$, and $2 p^{2} 3 d$; the correlation configurations are $2 s 3 p^{2}$ and $2 s 3 d^{2}$; and the Thomas-Fermi scaling parameters $\lambda_{n l}$ for the orbitals are $2.4(1 s)$, $1.37(2 s), 1.45(2 p), 1.4(3 s), 1.15(3 p)$, and 1.1(3d).

Calculations for photoionization cross sections were carried out twice, (1) for the partial cross sections leaving the core in the ground state and (2) for the total cross sections where the core can be in its ground or excited state depending on the energy. For each state, about 15,000 points of cross sections have been calculated to resolve the resonances. Calculations of partial cross sections require much longer CPU time due to reading and writing out of the isolated contributions of channels going only to the target ground state.

The Rydberg series of autoionizing resonances in $\sigma_{\mathrm{PI}}$ are resolved in detail with an effective quantum number mesh of 
TABLE 2

Radiative Decay Rates, $A_{j i}$, for Dipole Allowed Transitions from Various Excited Target States to the Ground State $2 s^{2} 2 p\left({ }^{2} P^{o}\right)$, of $\mathrm{Ar}$ XIV, ANd Comparison of the DR Collision Strength, $\langle\Omega(\mathrm{DR})\rangle$, with the Electron Impact Excitation Collision Strength, $\Omega$ (EIE), at $n=2$ Thresholds

\begin{tabular}{|c|c|c|c|}
\hline Target State & $\begin{array}{c}A_{f i} \\
\text { (a.u.) }\end{array}$ & $\Omega(\mathrm{EIE})$ & $\langle\Omega(\mathrm{DR})\rangle$ \\
\hline \multicolumn{4}{|c|}{$\Delta n=0$ Transitions } \\
\hline $2 s 2 p^{2}\left({ }^{2} D\right)$ & $7.46(-8)$ & 1.204 & 1.272 \\
\hline 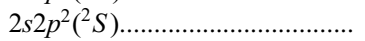 & $3.20(-7)$ & 0.453 & 0.509 \\
\hline $2 s 2 p^{2}\left({ }^{2} P\right)$ & $5.86(-7)$ & 2.180 & 2.162 \\
\hline \multicolumn{4}{|c|}{$\Delta n=1$ Transitions } \\
\hline $2 s^{2} 3 s\left({ }^{2} S\right)$ & $1.32(-5)$ & 0.059 & \\
\hline $2 s^{2} 3 d\left({ }^{2} D\right)$ & $7.97(-5)$ & 1.050 & \\
\hline $2 s 2 p 3 p\left({ }^{2} D\right)$ & $3.81(-5)$ & 0.526 & \\
\hline $2 s 2 p 3 p\left({ }^{2} P\right) \ldots \ldots \ldots \ldots \ldots \ldots \ldots \ldots \ldots$ & $3.33(-5)$ & 0.010 & \\
\hline $2 s 2 p 3 p\left({ }^{2} S\right)$ & $3.84(-5)$ & 0.019 & \\
\hline $2 s 2 p 3 p\left({ }^{2} P\right)$ & $9.98(-6)$ & 0.020 & \\
\hline $2 s 2 p 3 p\left({ }^{2} D\right)$ & $2.84(-6)$ & 0.008 & \\
\hline $2 s 2 p 3 p\left({ }^{2} S\right)$ & $5.44(-6)$ & 0.033 & \\
\hline $2 s 2 p 3 p\left({ }^{2} P\right)$ & $4.15(-6)$ & 0.003 & \\
\hline $2 s 2 p 3 p\left({ }^{2} D\right)$ & $7.98(-7)$ & 0.0009 & \\
\hline
\end{tabular}

$\Delta \nu=0.01$ up to $\nu=10$. This ensures that all resonance structures up to $\nu=10$ are resolved with a fine mesh of 100 points in each interval $(\nu, \nu+1)$. The resonances belonging to $n=3$ thresholds are resolved with a constant energy mesh. The resonance profiles decrease in width with effective quantum number as $\nu^{-3}$ relative to the threshold of convergence such that $\nu(E)=$ $z\left(E-E_{t}\right)^{-1 / 2}$ (in ryd), where $E_{t}$ is the target threshold energy and $E$ is the continuum electron energy. In the region below each target threshold, the narrow autoionizing resonances are averaged using the Gailitis resonance averaging procedure (e.g., Nahar \& Pradhan 1994). The photoionization cross sections at higher photoelectron energies, beyond the highest target threshold, are extrapolated as explained in Nahar \& Pradhan (1994).

The total DR cross sections in the region $n_{0}<\nu \leq \infty$ below each threshold are obtained for all regions with thresholds radiatively allowed to decay to the core state. STGFDR (Nahar \& Pradhan 1994) was employed to obtain $\Omega(\mathrm{DR})$. The transition probabilities ( $A$-values) for dipole allowed transitions to the target ground $2 s^{2} 2 p\left({ }^{2} P^{o}\right)$ state from ${ }^{2} D,{ }^{2} S$, and ${ }^{2} P$ states of the $n=2$ and 3 complexes are obtained from the atomic structure calculations using SUPERSTRUCTURE. These $A$-values are given in Table 2. It may be noted that radiative decay rates for $\Delta n=1$ are much stronger, over a magnitude higher, than those of $\Delta n=0$, indicating strong coupling effects. This was the reason for inclusion of $n=3$ states in the present work.

To compare the consistency of DR collision strengths, $\Omega(\mathrm{DR})$, electron impact excitation collision strengths, $\Omega$ (EIE), at various target thresholds are obtained in the close-coupling approximation. These values are given in Table 2 along with the $\langle\Omega(D R)\rangle$ peak at these thresholds. Comparison shows $\Omega(\mathrm{DR})$ is converging to $\Omega$ (EIE). We found that such convergence is not very obvious for the high-energy $n=3$ thresholds where $\langle\Omega(D R)\rangle$ becomes much weaker, as evident from the values of $\Omega$ (EIE).

The state-specific and total recombination rate coefficients were obtained using program RECOMB. The major contributions are from the photoionization cross sections and high- $n$ DR collision strengths. Contributions from energies beyond the highest target state to infinity were obtained using the numerical technique described in Nahar \& Pradhan (1994) and from the "top-up" as described in Nahar (1996b). The large amount of recombination data was processed by the program PRCOMG.

\section{RESULTS AND DISCUSSION}

The radiative inverse processes of photoionization and electron ion recombination of $\mathrm{Ar} \mathrm{XIII}+h \nu \leftrightarrow \mathrm{Ar} \mathrm{XIV}+e$ are studied in detail. Features of photoionization cross sections in the low-energy region of $n=2$ core thresholds were studied earlier (Nahar \& Pradhan 1992a). New features are revealed with a larger wavefunction affecting both the photoionization and recombination processes. Ar XIII is unique in showing multiple "DR" bumps in its recombination rates (Nahar 2001-2002 annual BAAS report by Pinsonneault \& Wing 2003).

The results were obtained twice, once using an $8 \mathrm{CC}$ wave function expansion, as used in the previous study (Nahar \& Pradhan 1992a), and then using a larger wavefunction expansion of 37 terms. The purpose was to study the effect of strong dipole transitions within the core in the high-energy region.

A total of 684 bound states $\left(N_{b}^{T}\right)$ of singlet, triplet, and quintet symmetries with $n \leq 10$ and $l \leq 9$ are obtained for Ar XIII. Large number of bound states is expected from an element with higher charge. However, the number of bound states remains the same with both 8 term and 37 term wavefunction expansions; that is, no new states are from with $n=3$ core states. Each state is assigned with an spectroscopic identification through quantum defect analysis. As explained in the computation section, there could be mismatches or uncertainties in identification for closely lying states, especially for highly excited states, owing to similar characteristics. The NIST compilation table lists 28 observed

TABLE 3

Comparison of Calculated Energies, $E_{c}$, with Measured Values, $E_{o}$ (KeLLY, NIST)

\begin{tabular}{|c|c|c|c|}
\hline Conf. & Term & $\begin{array}{c}E_{o} \\
\text { (ryd) }\end{array}$ & $\begin{array}{c}E_{c} \\
\text { (ryd) }\end{array}$ \\
\hline $2 s 22 p 2 \ldots \ldots \ldots \ldots \ldots$ & ${ }^{3} P$ & 50.191660 & 50.43 \\
\hline $2 s 22 p 2 \ldots \ldots \ldots \ldots \ldots \ldots \ldots \ldots \ldots \ldots \ldots \ldots$ & ${ }^{1} D$ & 49.558303 & 49.80 \\
\hline $2 s 22 p 2$ & ${ }^{1} S$ & 48.855637 & 49.09 \\
\hline $2 s 2 p 3 \ldots \ldots \ldots \ldots \ldots$ & ${ }^{5} S^{o}$ & 48.273847 & 48.49 \\
\hline $2 s 2 p 3 \ldots \ldots \ldots \ldots \ldots \ldots \ldots \ldots$ & ${ }^{3} D^{o}$ & 46.474028 & 46.66 \\
\hline $2 s 2 p 3 \ldots \ldots \ldots \ldots$ & ${ }^{3} P^{o}$ & 45.808361 & 46.00 \\
\hline $2 s 2 p 3$ & ${ }^{1} D^{o}$ & 44.629872 & 44.79 \\
\hline $2 s 2 p 3$ & ${ }^{3} S^{o}$ & 44.603992 & 44.74 \\
\hline 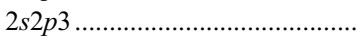 & ${ }^{1} P^{o}$ & 43.966379 & 44.13 \\
\hline $2 s 22 p 3 s$ & ${ }^{3} P^{o}$ & 21.597007 & 21.88 \\
\hline $2 s 22 p 3 s$ & ${ }^{1} P^{o}$ & 21.381077 & 21.69 \\
\hline $2 s 22 p 3 d \ldots \ldots \ldots$ & ${ }^{3} F^{o}$ & 19.385940 & 19.64 \\
\hline $2 s 22 p 3 d \ldots \ldots \ldots \ldots \ldots \ldots \ldots \ldots \ldots \ldots$ & ${ }^{1} D^{o}$ & 19.398160 & 19.65 \\
\hline $2 s 22 p 3 d \ldots \ldots \ldots \ldots \ldots \ldots \ldots \ldots$ & ${ }^{3} D^{o}$ & 19.164663 & 19.40 \\
\hline $2 s 22 p 3 d \ldots$. & ${ }^{3} P^{o}$ & 19.044548 & 19.34 \\
\hline $2 s 22 p 3 d \ldots \ldots \ldots \ldots$ & ${ }^{1} P^{o}$ & 18.740225 & 19.00 \\
\hline $2 s 22 p 3 d \ldots \ldots \ldots \ldots$ & ${ }^{1} F^{o}$ & 18.735669 & 19.00 \\
\hline $2 s 2 p 23 p$ & ${ }^{3} D^{o}$ & 17.125824 & 16.82 \\
\hline $2 s 2 p 2(4 P) 3 d \ldots \ldots \ldots \ldots \ldots \ldots \ldots \ldots$ & ${ }^{5} P *$ & 17.099291 & 17.29 \\
\hline $2 s 2 p 23 p$ & ${ }^{1} D^{o}$ & 16.019181 & 15.24 \\
\hline $2 s 2 p 23 p$ & ${ }^{3} P^{o}$ & 15.784439 & 15.87 \\
\hline $2 s 2 p 23 p$ & ${ }^{1} P^{o}$ & 15.580862 & 15.81 \\
\hline $2 s 2 p 23 p$ & ${ }^{3} S^{o}$ & 15.563001 & 15.54 \\
\hline $2 s 22 p 4 s$ & ${ }^{1} P^{o}$ & 11.826441 & 11.68 \\
\hline $2 s 22 p 4 s$ & ${ }^{3} P^{o}$ & 11.579852 & 11.63 \\
\hline 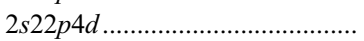 & ${ }^{1} P^{o}$ & 10.773927 & 10.66 \\
\hline $2 s 22 p 4 d \ldots \ldots \ldots \ldots$ & ${ }^{1} D^{o}$ & 10.598964 & 10.84 \\
\hline $2 s 22 p 4 d$ & ${ }^{3} P^{o}$ & 10.564700 & 10.78 \\
\hline
\end{tabular}




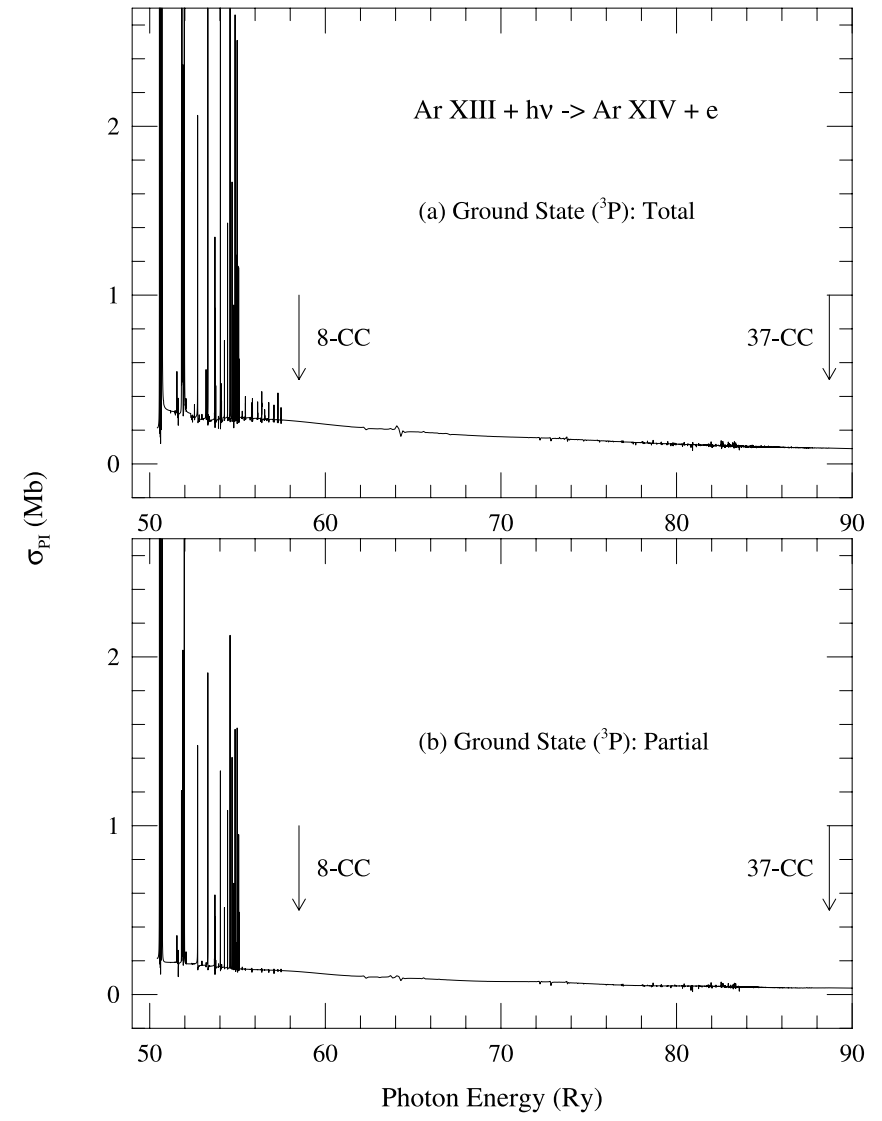

FIG. 1.-Photoionization cross sections, $\sigma_{\mathrm{PI}}$, of the ground $2 s^{2} 2 p^{2}\left({ }^{3} P\right)$ state of Ar XIII: (a) total-leaving the core ion in ground and various excited states; (b) partial-leaving the core ion in the ground state. The arrows point the photon energies at the highest core threshold for the $8 \mathrm{CC}$ and for the $37 \mathrm{CC}$ wavefunction expansion. The prominent resonances in the low-energy region belong to core thresholds of the $n=2$ complex.

states, which are compared with the calculated energies in Table 3. The agreement between the calculated and observed energies is within $2 \%$ for 26 states. The largest difference is about $5 \%$ with $2 s 2 p^{2} 3 p\left({ }^{1} D^{o}\right)$ state.

Details of photoionization cross sections, recombination cross sections and rate coefficients are presented in separate subsections below.

\subsection{Total and Partial Photoionization Cross Sections}

Total photoionization cross sections $\left(\sigma_{\mathrm{PI}}\right)$ leaving the ion in various excited states are presented for all 684 bound states. However, partial photoionization cross sections leaving core into the ground $2 s^{2} 2 p\left({ }^{2} P^{o}\right)$ state are obtained for the 561 states of singlet and triplet symmetries. Singlets and triplets couple to the core ground state, ${ }^{2} P^{o}$, and contribute to the total recombination rate coefficients.

Photoionization cross sections of the ground and equivalent electron states appear to be unaffected by extension of the wavefunction. However, considerable changes can be seen in the excited state photoionization cross sections. The $n=3$ core states introduce extensive resonances in the high-energy region via strong coupling effects. In many cases, these resonances are considerably more prominent than those from the $n=2$ complex.

Figure 1 presents the total (Fig. 1a) and partial (Fig. 1b) $\sigma_{\mathrm{PI}}$ for the $2 s^{2} 2 p^{2}\left({ }^{3} P\right)$ ground state of Ar XIII. The differences between total and partial $\sigma_{\mathrm{PI}}$ are in (1) missing some resonances

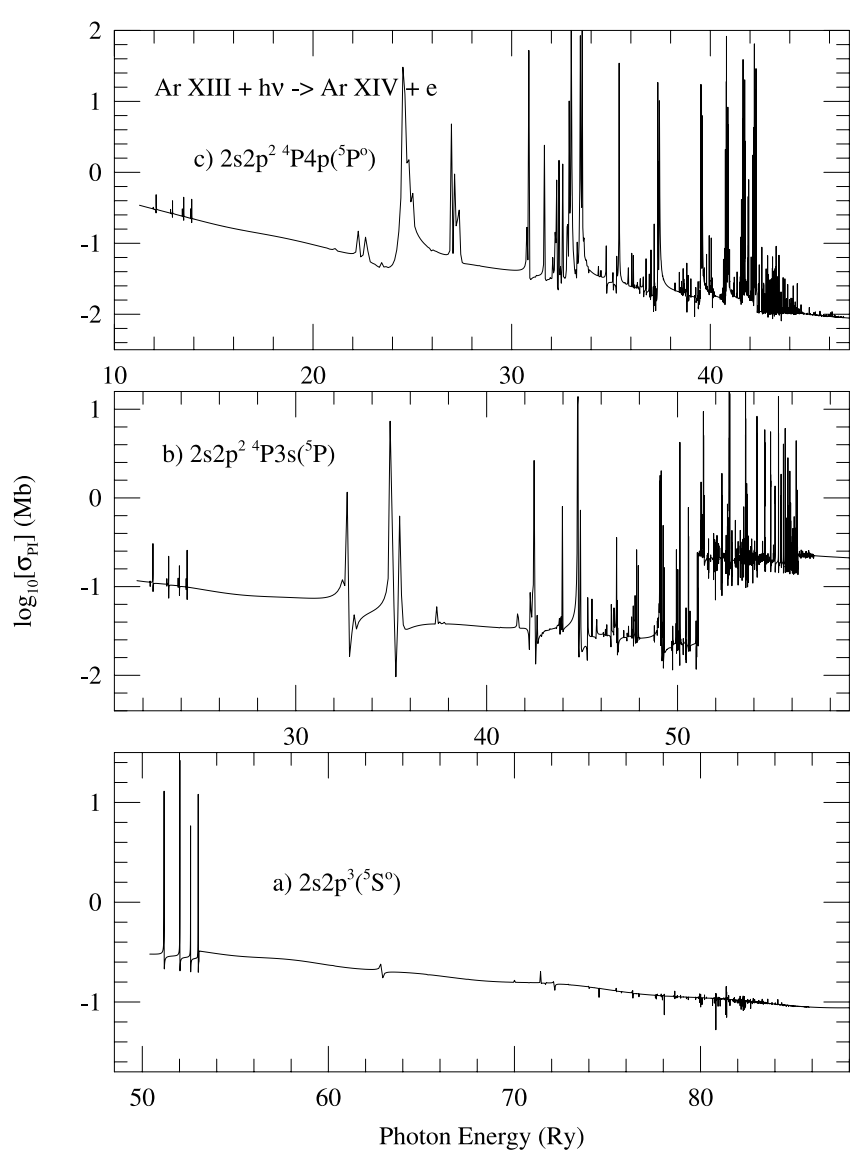

FIG. 2.-Photoionization cross sections of excited quintet states of Ar XIII illustrating coupling effects of $n=3$ core thresholds: while no significant effect is seen in $\sigma_{\mathrm{PI}}$ for the $(a)$ metastable $2 s 2 p^{3}\left({ }^{5} S^{o}\right)$ state, extensive and much enhanced resonant features are generated for the valence electron states, (b) $2 s 2 p^{2} 4 P 3 s\left({ }^{5} P\right)$, and (c) $2 s 2 p^{2}{ }^{4} P 4 p\left({ }^{5} P^{o}\right)$.

and (2) lower background at higher energies in partial $\sigma_{\mathrm{PI}}$ caused by omission of contributions from channels with excited cores.

The arrows in the panels point to the photon energies at the highest core threshold for the $8 \mathrm{CC}$ and for the $37 \mathrm{CC}$ wavefunction expansions. Much of the resonances are in the lower energy region, from ionization threshold to about 8 ryd above in both panels, and correspond to Rydberg series of autoionizing states belonging to $n=2$ core thresholds. No significant contribution comes from $n=3$ core thresholds as beyond $n=2$ thresholds, the cross section decays slowly with a smooth background except for some very small and weak resonances. The resonances of $n=2$ thresholds can affect both photoionization and recombination rates in the lower temperature region. The resonance positions in the present work should be more accurate than those by Nahar \& Pradhan (1992a) since the excited threshold energies, where the resonances converge, correspond to the more accurate measured values.

Figure 2 presents photoionization cross sections of three excited quintet states of $\mathrm{Ar}$ XIII: the metastable $2 s 2 p^{3}\left({ }^{5} S^{o}\right)$ state (Fig. 2a) and the valence electron $2 s 2 p^{2}{ }^{4} P 3 s\left({ }^{5} P\right)$ and $2 s 2 p^{2}{ }^{4} P 4 p\left({ }^{5} P^{o}\right)$ states (Figs. $2 b$ and $2 c$ ). Similar to the ground state, the low-energy resonances of the metastable ${ }^{5} S^{o}$ state in Figure $2 a$ belong to $n=2$ thresholds, while only some weak resonances of $n=3$ thresholds scantly fill up the background cross section. In contrast, for ${ }^{5} P$ and ${ }^{5} P^{o}$ states the resonances due to $n=3$ thresholds at energies beyond those of $n=2$ thresholds (beyond 8 ryd from the ionization threshold) are much stronger 


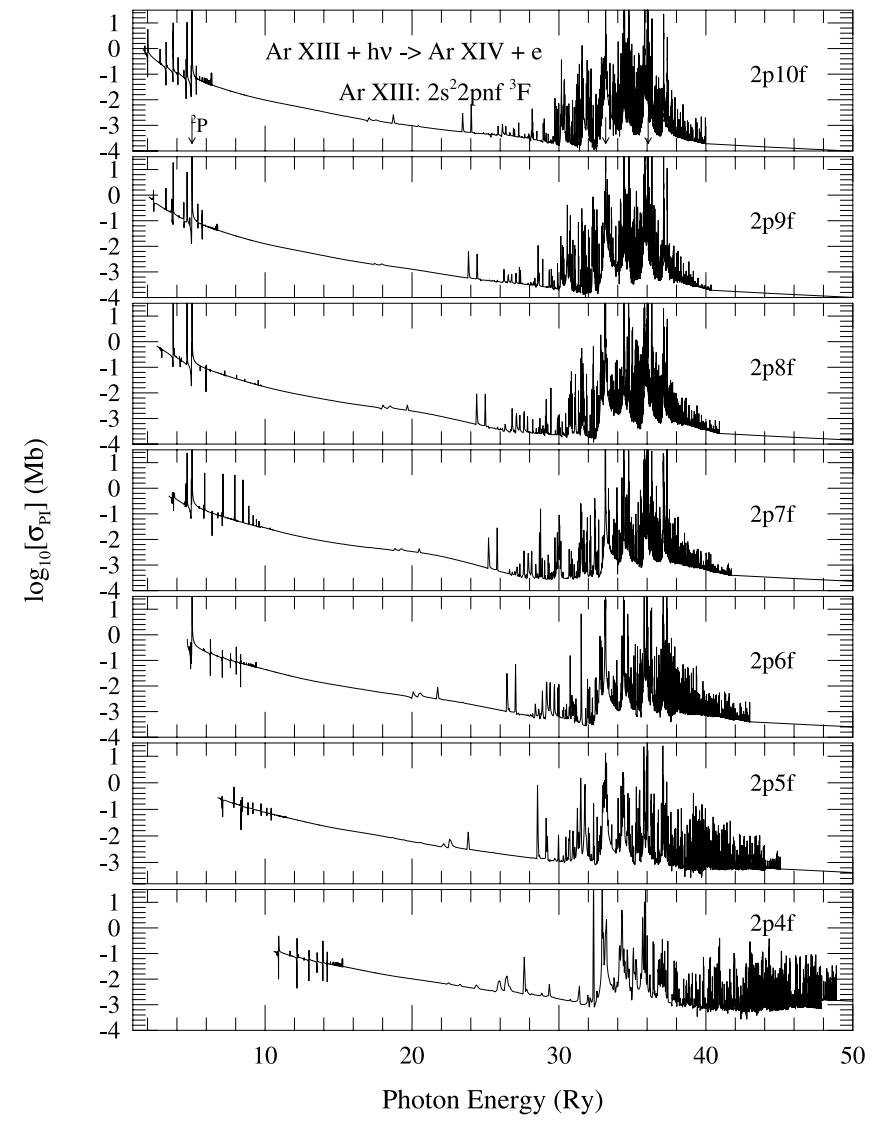

Fig. 3.- Photoionization cross sections of the Rydberg series of states, $2 s^{2} 2 p n f\left({ }^{3} F\right), 4 f \leq n f \leq 10 f$, of Ar XIII illustrating the large photoexcitationof-core (PEC) resonances at high photon energies. PECs are manifested at excited core thresholds, radiatively allowed to decay to $\mathrm{Ar}$ XIV (core) ground state $2 s^{2} 2 p\left({ }^{2} P^{o}\right)$. The arrows indicate a few PEC positions, one at ${ }^{2} P$ state of $n=2$, and two at ${ }^{2} D$ and ${ }^{2} P$ states of $n=3$ thresholds showing much stronger PEC resonances at $n=3$ thresholds in the photon energy region of $31-38$ ryd.

than those of $n=2$ thresholds. The background cross section may also be enhanced considerably, such as for the ${ }^{5} P$ state, at $n=3$ thresholds (at about 51.5 ryd).

At photon energies equal to that for dipole allowed transitions in the core ion, the core is excited, while the outer Rydberg electron remains a "spectator," weakly interacting with the core ion. This process, essentially the inverse of DR, introduces a relatively wide and pronounced resonance, known as the PEC (photoexcitation-of-core) resonance (Yu \& Seaton 1987), in the photoionization cross section. Figure 3 illustrates such PEC resonances in $\sigma_{\mathrm{PI}}$ of the Rydberg series of states, $2 s^{2} 2 p n f\left({ }^{3} F\right)$, with $4 f \leq n f \leq 10 f$, of $\mathrm{Ar}$ XIII. The ionization threshold for $\sigma_{\mathrm{PI}}$ moves toward lower photon energies with higher excited states. The thin set of resonances at lower energies near the ionization threshold for each state belong to $n=2$ core states, while the dense and enhanced set of resonances at higher energies belong to $n=3$ core states. These resonances at higher energies contribute more dominantly than those of $n=2$ states to photoionization rates. The arrows in the top panel of the figure point out a few PEC resonances at ${ }^{2} P$ of $n=2,{ }^{2} D$, and ${ }^{2} P$ of $n=3$ thresholds, allowed for dipole transitions to the core ground state $2 s^{2} 2 p\left({ }^{2} P^{o}\right)$. PEC resonances are observed in cross sections for excited valence electron states only since they entail core excitations and demonstrate the departure from near-hydrogenic behavior of cross sections of excited states.

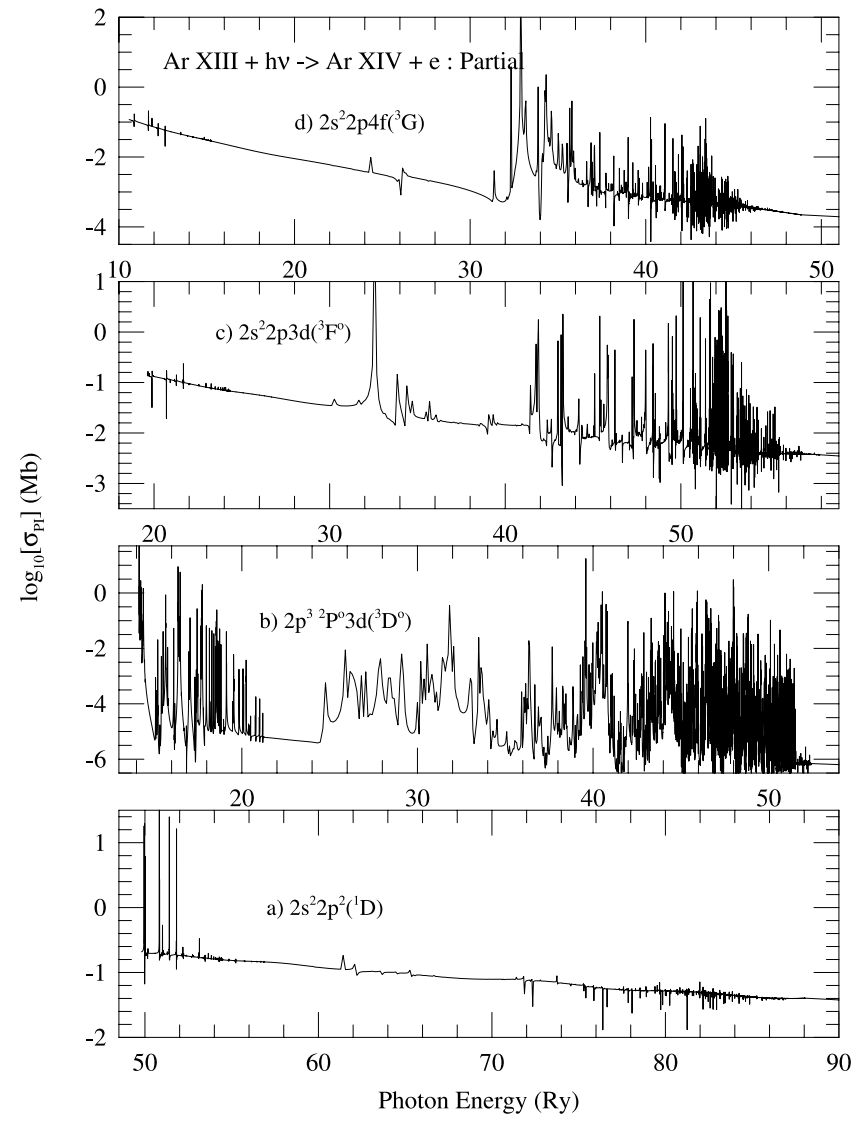

FIG. 4.-Partial photoionization cross sections of some states contributing dominantly to the total $\alpha_{\mathrm{RC}}$ of $\mathrm{Ar}$ XIII: $(a) 2 s^{2} 2 p^{2}\left({ }^{1} D\right),(b) 2 p^{3}{ }^{2} P^{o} 3 d\left({ }^{3} D^{o}\right)$, (c) $2 s^{2} 2 p 3 d\left({ }^{3} F^{o}\right)$, and $(d) 2 s^{2} 2 p 4 f\left({ }^{3} G\right)$. The dominance is determined by the enhanced background cross sections the and positions of strong resonant features.

Detailed features of partial photoionization cross sections for a few states, in relevance to the recombination rates, are illustrated in Figure 4. These, $2 s^{2} 2 p^{2}\left({ }^{1} D\right)$ (Fig. $\left.4 a\right), 2 p^{32} P^{o} 3 d\left({ }^{3} D^{o}\right)$ (Fig. $4 b$ ), $2 s^{2} 2 p 3 d\left({ }^{3} F^{o}\right)$ (Fig. $4 c$ ), and $2 s^{2} 2 p 4 f\left({ }^{3} G\right)$ (Fig. $4 d$ ), are among the dominant contributors to the total recombination rate coefficients of $\mathrm{Ar}$ XIII at various temperature regions. The equivalent electron states, such as $2 s^{2} 2 p^{2}\left({ }^{1} D\right)$ of ground configuration in Figure $4 a$, have relatively higher background cross sections that decay slowly with energies, and hence contribute considerably to $\alpha_{R}(T)$ for a wide range of temperature. In Figure $4 b,{ }^{3} D^{o}$ is considerably dominant at lower temperatures because of its high-resonance peaks in the near threshold region. The other two states, ${ }^{3} F^{o}$ in Figure $4 c$ and ${ }^{3} G$ in Figure $4 d$, are dominant at temperatures where the resonant features are enhanced.

\subsection{DR Collision Strengths and Recombination Cross Sections}

The total dielectronic recombination collision strengths, $\Omega(\mathrm{DR})$, of highly excited group B states, $10 \leq n \leq \infty$, for $e+\operatorname{Ar}$ XIV $\rightarrow$ Ar XIII are obtained both in detailed form with resonances and in resonance-averaged form. The value of $\Omega(\mathrm{DR})$ in the energy regions below the excited target thresholds ${ }^{2} D,{ }^{2} S$, and ${ }^{2} P$ of the $n=2$ complex is presented in Figure 5, where the dotted curve represents the detailed form and the solid curve represents the resonance-averaged one. The figure has two panels: the lower one shows the expanded and detailed structures of $\Omega(\mathrm{DR})$, and the upper one shows the peak values, particularly the averaged $\Omega(\mathrm{DR})$, as the resonances converge 


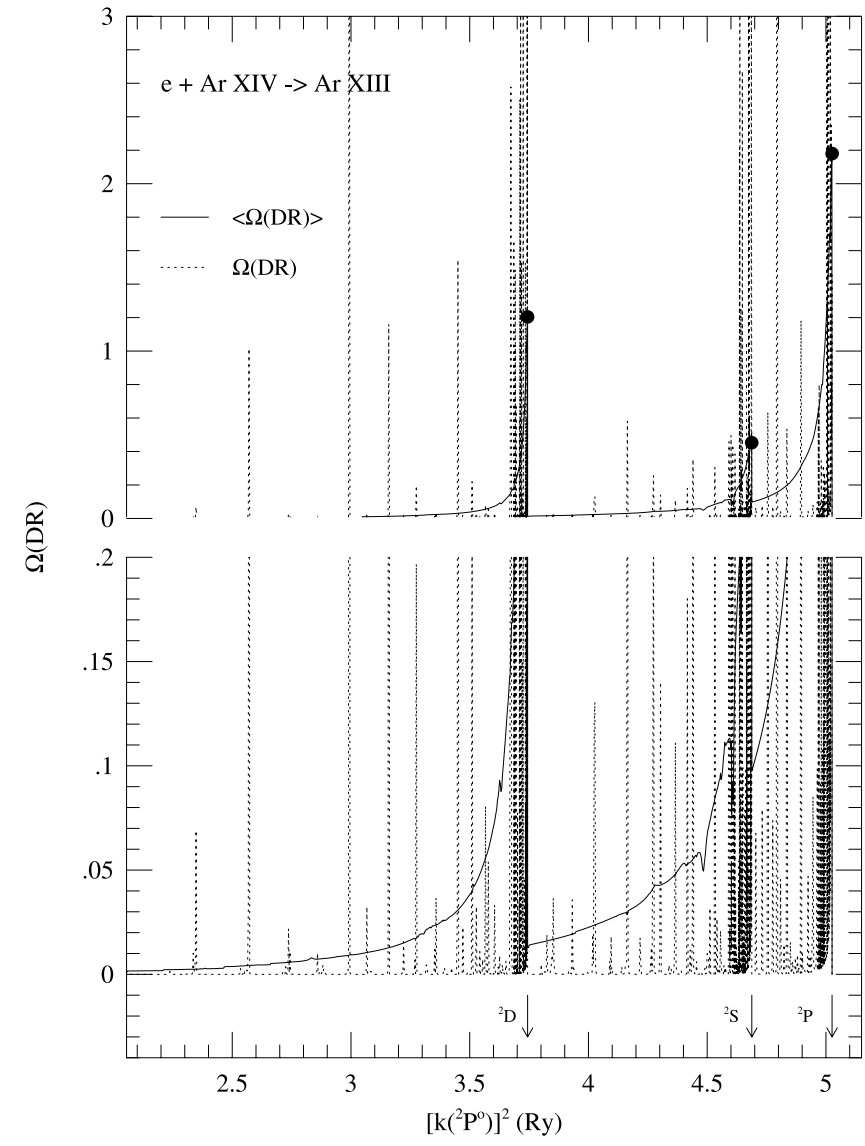

FIG. 5.-DR collision strengths $\Omega$ (DR) for Ar XIII: detailed with resonances (dotted curve), and averaged over resonances (solid curve), in the regions below and at the excited core thresholds ${ }^{2} D,{ }^{2} S$, and ${ }^{2} P$ of the $n=2$ complex (positions are specified by arrows). Lower panel shows expanded form of $\Omega(D R)$. The filled circles are the calculated electron impact excitation (EIE) collision strength, $\Omega($ EIE), at the thresholds.

on to their respective thresholds. The DR features show that the density of resonances increases with effective quantum number, while the background rises as $\nu^{3}$, indicating an increase in electron capture via the DR process as the energy approaches the threshold. At the threshold DR drops to zero as the core ion is now excited, i.e., the trapped electron flux due to DR is released into the scattering electron-impact excitation (EIE) channels. The nonzero contributions in the figure at the ${ }^{2} S$ threshold are from the next higher ${ }^{2} P$ channels.

The conservation of (photon+electron) flux requires that the peak of the resonance-averaged $\Omega(D R)$ at a threshold should equal the collision strength for electron impact excitation, $\Omega$ (EIE). The filled circles in Figure 5 are the calculated $\Omega$ (EIE) at the thresholds and are seen to match the $\langle\Omega(D R)\rangle$ peak. The numerical values are given in Table 2 showing the good agreement. The DR contributions of high- $n$ states below $n=3$ core thresholds become much weaker, while the contributions from lower $n$ bound states of group A become important through photoionization cross sections. Table 2 shows weak core excitations for $n=3$ thresholds.

The unified electron-ion recombination cross sections, $\sigma_{\mathrm{RC}}$, can be obtained from the sum of photorecombination cross sections of low- $n$ bound states and DR cross sections of high- $n$ states. Figure $6 a$ presents $\sigma_{\mathrm{RC}}$ from about zero photoelectron energy to the highest core threshold beyond which no resonant structure is expected. The low-energy cross sections are dominated by Rydberg series of resonances belonging to $n=2$
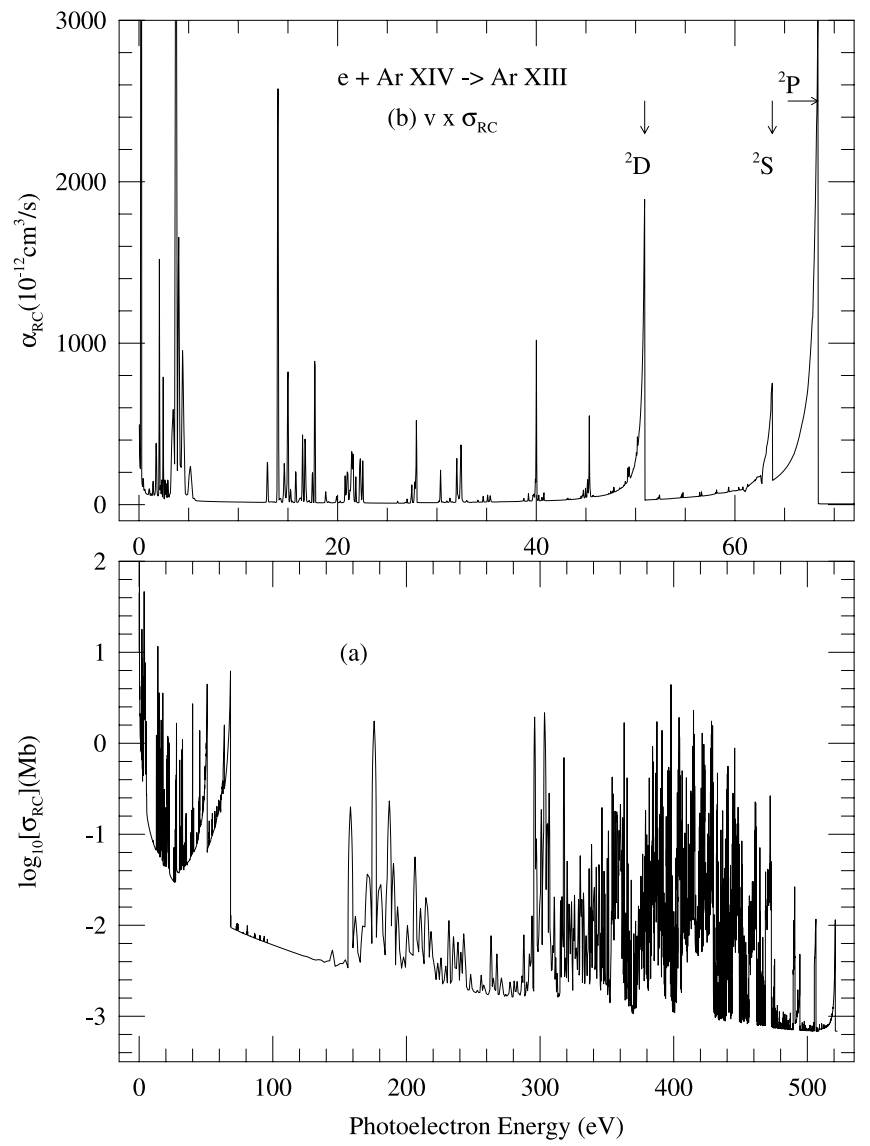

FIG. 6.-(a) Detailed unified $\sigma_{\mathrm{RC}}$ in the energy range of the $n=2$ and 3 complexes; $(b)$ detailed recombination rate coefficient $\left(v \sigma_{\mathrm{RC}}\right)$ in the energy range of the $n=2$ complex. The dominant DR contributions can be seen below and at the core thresholds, ${ }^{2} D,{ }^{2} S$, and ${ }^{2} P$.

thresholds, especially up to the energy $(68 \mathrm{eV})$ of $2 s 2 p^{2}{ }^{2} P$, the highest $n=2$ core state accessible from the ground state via dipole transition. The peak at this threshold is mainly from DR contribution. Although the background is about zero for the rest of the energy range, the contributions are mainly from the resonant photorecombination cross sections at higher energies of Ar XIII bound states with $n \leq 10$. The high- $n$ DR makes a relatively small contribution.

The recombination rate, $\alpha_{\mathrm{RC}}=v \sigma_{\mathrm{RC}}$, at various photoelectron energies is a measurable quantity at storage rings (e.g., Savin et al. 2003). Figure $6 b$ presents the recombination rates, $\alpha_{\mathrm{RC}}(\mathrm{eV})$, up to core threshold $2 s 2 p^{2}{ }^{2} P$. These rates should be convolved with experimental monochromatic bandwidth for direct comparison. The rate $\alpha_{\mathrm{RC}}$ for Ar XIII is dominated by the near threshold resonances. As the energy approaches the $n=2$ core thresholds allowed for radiative decays, DR dominates. The DR peaks are indicated by arrows at the three thresholds, $2 s 2 p^{2}\left({ }^{2} D,{ }^{2} S,{ }^{2} P\right)$. Recently, Savin et al. (2003) have measured such rates of a similar carbon-like ion, Fe XXI, in the lowenergy region. The present work is expected to motivate such experiments for Ar XIII. While present results are in LS coupling, a more resolved spectrum with fine-structure effects is expected to be observed in the experiment as in the case for Fe XXI.

\subsection{Total and State-specific Recombination Rate Coefficients}

The total recombination rate coefficients $\alpha_{R}(T)$ for $(e+$ Ar XIV) $\rightarrow$ Ar XIII are presented for a wide range of temperatures, 
TABLE 4

Total Recombination Rate Coefficients, $\alpha_{R}(T)$ FOR $e+\operatorname{Ar} \mathrm{XIV} \rightarrow \operatorname{Ar} \mathrm{XIII}$ in Temperature Range, $1 \mathrm{~K} \leq \log _{10} T \leq 9 \mathrm{~K}$

\begin{tabular}{|c|c|}
\hline $\begin{array}{c}\log _{10} T \\
(\mathrm{~K})\end{array}$ & $\begin{array}{c}\alpha_{R} \\
\left(\mathrm{~cm}^{3} \mathrm{~s}^{-1}\right)\end{array}$ \\
\hline $1.0 \ldots \ldots$ & $9.20 \mathrm{E}-09$ \\
\hline $1.1 \ldots \ldots \ldots \ldots \ldots \ldots \ldots$ & $8.16 \mathrm{E}-09$ \\
\hline $1.2 \ldots \ldots \ldots \ldots \ldots \ldots \ldots \ldots$ & $7.23 \mathrm{E}-09$ \\
\hline 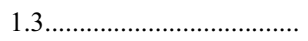 & $6.40 \mathrm{E}-09$ \\
\hline 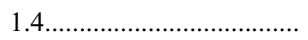 & $5.66 \mathrm{E}-09$ \\
\hline 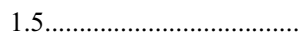 & $5.01 \mathrm{E}-09$ \\
\hline $1.6 \ldots \ldots \ldots \ldots \ldots \ldots \ldots \ldots \ldots . .$. & $4.42 \mathrm{E}-09$ \\
\hline 1.7 & $3.90 \mathrm{E}-09$ \\
\hline 1.8 & $3.43 \mathrm{E}-09$ \\
\hline 1.9 & $3.02 \mathrm{E}-09$ \\
\hline 2.0 & $2.65 \mathrm{E}-09$ \\
\hline $2.1 \ldots$ & $2.33 \mathrm{E}-09$ \\
\hline 2.2 & $2.04 \mathrm{E}-09$ \\
\hline 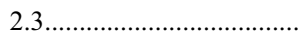 & $1.78 \mathrm{E}-09$ \\
\hline $2.4 \ldots$ & $1.56 \mathrm{E}-09$ \\
\hline $2.5 \ldots \ldots$ & $1.39 \mathrm{E}-09$ \\
\hline $2.6 \ldots \ldots \ldots . .$. & $1.31 \mathrm{E}-09$ \\
\hline 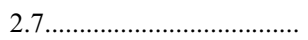 & $1.35 \mathrm{E}-09$ \\
\hline 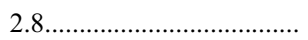 & $1.52 \mathrm{E}-09$ \\
\hline $2.9 \ldots \ldots \ldots-1$ & $1.76 \mathrm{E}-09$ \\
\hline $3.0 \ldots \ldots \ldots-1, \ldots$ & $1.99 \mathrm{E}-09$ \\
\hline 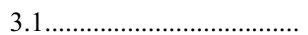 & $2.14 \mathrm{E}-09$ \\
\hline 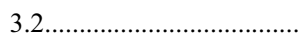 & $2.16 \mathrm{E}-09$ \\
\hline $3.3 \ldots$ & $2.05 \mathrm{E}-09$ \\
\hline 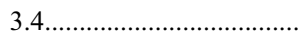 & $1.86 \mathrm{E}-09$ \\
\hline 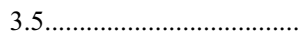 & $1.61 \mathrm{E}-09$ \\
\hline 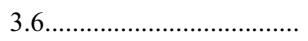 & $1.36 \mathrm{E}-09$ \\
\hline 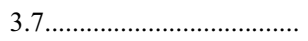 & $1.12 \mathrm{E}-09$ \\
\hline 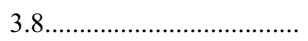 & $9.12 \mathrm{E}-10$ \\
\hline 3.9.............................. & $7.49 \mathrm{E}-10$ \\
\hline 4.0 & $6.30 \mathrm{E}-10$ \\
\hline 4.1 & $5.48 \mathrm{E}-10$ \\
\hline 4.2 & $4.92 \mathrm{E}-10$ \\
\hline $4.3 \ldots$ & $4.47 \mathrm{E}-10$ \\
\hline 4.4 & $4.05 \mathrm{E}-10$ \\
\hline 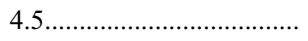 & $3.62 \mathrm{E}-10$ \\
\hline 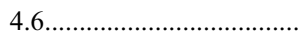 & $3.17 \mathrm{E}-10$ \\
\hline 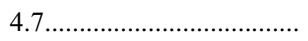 & $2.74 \mathrm{E}-10$ \\
\hline . & $2.34 \mathrm{E}-10$ \\
\hline $4.9 \ldots \ldots \ldots$ & $1.98 \mathrm{E}-10$ \\
\hline 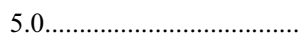 & $1.68 \mathrm{E}-10$ \\
\hline 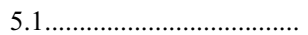 & $1.43 \mathrm{E}-10$ \\
\hline $5.2 \ldots \ldots \ldots-1, \ldots$ & $1.24 \mathrm{E}-10$ \\
\hline $5.3 \ldots \ldots-1-1$ & $1.09 \mathrm{E}-10$ \\
\hline 5.4 & $9.79 \mathrm{E}-11$ \\
\hline $5.5 \ldots$ & $8.79 \mathrm{E}-11$ \\
\hline $5.6 \ldots \ldots \ldots \ldots \ldots \ldots \ldots \ldots \ldots \ldots . .$. & $7.88 \mathrm{E}-11$ \\
\hline 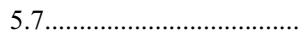 & $7.04 \mathrm{E}-11$ \\
\hline 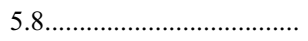 & $6.29 \mathrm{E}-11$ \\
\hline 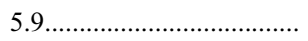 & $5.67 \mathrm{E}-11$ \\
\hline 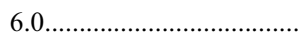 & $5.19 \mathrm{E}-11$ \\
\hline $6.1 \ldots \ldots \ldots-1$ & $4.81 \mathrm{E}-11$ \\
\hline 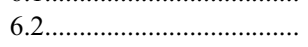 & $4.48 \mathrm{E}-11$ \\
\hline 6.3............................... & $4.12 \mathrm{E}-11$ \\
\hline 6.4 & $3.71 \mathrm{E}-11$ \\
\hline 6.5 & $3.25 \mathrm{E}-11$ \\
\hline 6.6 & $2.77 \mathrm{E}-11$ \\
\hline 6.7 & $2.29 \mathrm{E}-11$ \\
\hline 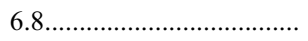 & $1.84 \mathrm{E}-11$ \\
\hline 6.9 & $1.45 \mathrm{E}-11$ \\
\hline 7.0 & $1.12 \mathrm{E}-11$ \\
\hline
\end{tabular}

TABLE 4-Continued

\begin{tabular}{|c|c|}
\hline $\begin{array}{c}\log _{10} T \\
(\mathrm{~K})\end{array}$ & $\begin{array}{c}\alpha_{R} \\
\left(\mathrm{~cm}^{3} \mathrm{~s}^{-1}\right)\end{array}$ \\
\hline 7.1 & $8.57 \mathrm{E}-12$ \\
\hline 7.2 & $6.46 \mathrm{E}-12$ \\
\hline 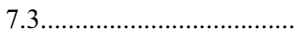 & $4.81 \mathrm{E}-12$ \\
\hline 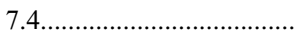 & $3.56 \mathrm{E}-12$ \\
\hline 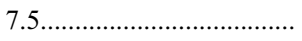 & $2.62 \mathrm{E}-12$ \\
\hline 7.6............................ & $1.92 \mathrm{E}-12$ \\
\hline $7.7 \ldots \ldots$ & $1.40 \mathrm{E}-12$ \\
\hline 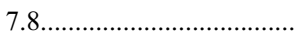 & $9.93 \mathrm{E}-13$ \\
\hline 7.9 . & $7.19 \mathrm{E}-13$ \\
\hline $8.0 \ldots \ldots \ldots-1, \ldots$ & $5.19 \mathrm{E}-13$ \\
\hline $8.1 \ldots \ldots \ldots \ldots \ldots \ldots \ldots \ldots .$. & $3.73 \mathrm{E}-13$ \\
\hline 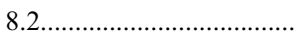 & $2.68 \mathrm{E}-13$ \\
\hline 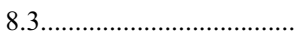 & $1.93 \mathrm{E}-13$ \\
\hline 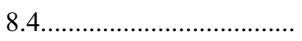 & $1.39 \mathrm{E}-13$ \\
\hline 8.5 & $9.98 \mathrm{E}-14$ \\
\hline 8.6 & $7.18 \mathrm{E}-14$ \\
\hline 8.7 & $5.17 \mathrm{E}-14$ \\
\hline 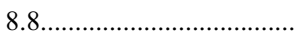 & $3.72 \mathrm{E}-14$ \\
\hline 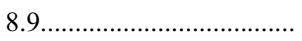 & $2.68 \mathrm{E}-14$ \\
\hline 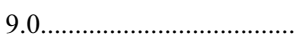 & $1.93 \mathrm{E}-14$ \\
\hline
\end{tabular}

$1 \leq \log _{10}(T) \leq 9$, at a fine temperature mesh of $\Delta \log _{10} T=0.1$. The numerical values are presented in Table 4 .

A unique feature of multiple DR "bumps," four in total, which has not been seen with any other ions, is found in the total recombination rate coefficients. These bumps enhance the total $\alpha_{R}(T)$ considerably, as illustrated in Figure 7 alongside comparison with previous calculations. The lower panel of Figure 7 presents recombination rate coefficients for the entire temperature range and the upper two panels detail the "DR bumps." In the lower panel, the solid curve represents total unified $\alpha_{R}$ using the $37 \mathrm{CC}$ expansion and the dotted curve represents the same but using the $8 \mathrm{CC}$ expansion. The RR rate ceofficients (dashed curve) were obtained by Aldrovandi \& Pequignot (1974) using photoionization cross sections in central field for the ground state and hydrogenic approximation by Seaton (1959) for excited states. The DR rate coefficients by Shull \& van Steenberg (1982, chain-dashed curve) were obtained using the Burgess (1965) general formula; the coefficients by $\mathrm{Gu}(2003)$ and by Altun et al. (2004, dot-chained curve) were obtained using isolated resonance approximation with distorted waves.

In the upper panels of Figure 7, the "DR bumps" are indicated by arrows, four in the $37 \mathrm{CC}$ calculations and three in the $8 \mathrm{CC}$ calculations. The first two bumps at around 2000 and $30,000 \mathrm{~K}$ in the total $\alpha_{R}(T)$ for $\mathrm{Ar}$ XIII are due to autoionizing resonances in the near threshold region of photoionization cross sections of states, such as $2 s 2 p^{3}\left({ }^{3} D^{o}\right), 2 p^{3} 3 s\left({ }^{3} D^{o}\right)$ for the first bump and $2 s 2 p^{3}\left({ }^{3} D^{o}\right), 2 s^{2} 2 p^{2}\left({ }^{3} P\right)$ for the second bump, as illustrated in Figure 5. The second bump can be seen in the DR rates by $\mathrm{Gu}$ (2003) (in graphical presentation) and by Altun et al. (2004). The third bump (more distinct in $8 \mathrm{CC}$ rates) at high temperature at around $2.5 \times 10^{5} \mathrm{~K}$ is from dominance of DR near $n=2$ core thresholds and is seen in all three previous DR calculations, by Shull \& van Steenberg (1982), Gu (2003), and Altun et al. (2004). Resonances due to stronger radiative decay rates of $n=3$ core states have introduced another DR bump at very high temperature around $2 \times 10^{6} \mathrm{~K}$, almost flattening out the third one. This bump is also seen in the DR rates 


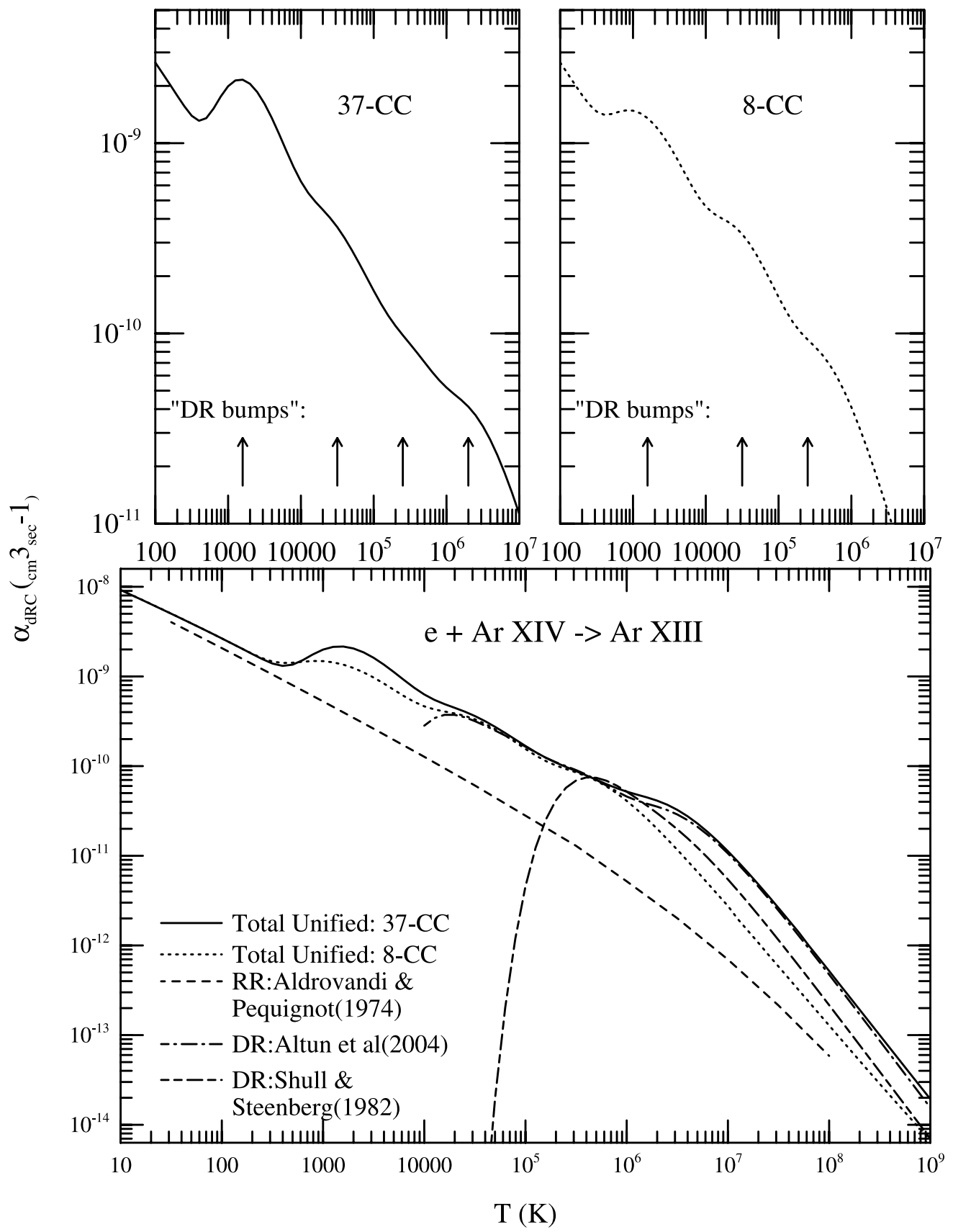

FIG. 7.- Recombination rate coefficients $\alpha_{R}(T)$ of $(e+\mathrm{Ar}$ XIV $) \rightarrow \operatorname{Ar}$ XIII. Curves in the lower panel: solid curve, total unified rate coefficients using the $37 \mathrm{CC}$ expansion; dotted curve, same but using the 8 CC expansion; dashed curve, RR rate coefficients of Aldrovandi \& Pequignot (1974); chain-dashed curve, DR rate coefficients by Shull \& Steenberg (1982); dot-dashed curve, DR rate coefficients by Altun et al. (2004). The upper two panels show the "DR bumps" (arrows), four in $37 \mathrm{CC}$ calculations and three in $8 \mathrm{CC}$ calculations.

of $\mathrm{Gu}(2003)$ and of Altun et al. (2004). Typically, the total $\alpha_{R}(T)$, dominated by RR at very low temperature decreases with temperature until at high $T$, where it rises because of the dominance of DR, which is followed by a smooth decay. A lowtemperature "bump" in $\alpha_{R}(T)$ due to near threshold resonances exists for many ions, e.g., for O III (Paper I). However, the presence of four bumps has been seen only for Ar XIII so far. The new features indicate that the total rate does not necessarily have a smooth transition from RR to DR with temperature.

No DR bump is seen in the previous work by Aldrovandi \& Pequignot (dashed curve) as no autoionizing resonances were included in their cross sections. However, at very low temperature their RR rate coefficient almost merges with the present rates. This is expected at very low temperature since the electrons are not energetic enough to introduce a doubly excited state for DR. The high-temperature DR rates (dash-chained curve) by Shull \& van Steenberg (1982) show fair agreement with the present results at $n=2$ thresholds. Their rates do not show the prominent enhancement from the $n=3$ states, indicating transitions from these states were not included in the Burgess formula. The present total $\alpha_{R}(T)$-values show very good agreement with the DR rates of Altun et al. (dot-dashed curve) from about $2 \times 10^{3}$ to $10^{9} \mathrm{~K}$ conceding dominance of DR over RR at high temperature. However, below this temperature range, their DR rate drops down as no RR was included in their calculations.

State-specific recombination rate coefficients for individual bound states of Ar XIII are presented for 561 low- $n$ group A 
TABLE 5-Continued

TABLE 5

State-specific Recombination Rate Coefficients of the 20 Dominant States of Ar xiII at Temperatures $T=1 \times 10^{3}, 1 \times 10^{4}$, $1 \times 10^{5}$, AND $1 \times 10^{6} \mathrm{~K}$ IN ORder OF TheIr Contributions to the Total $\alpha_{R}(T)$

\begin{tabular}{|c|c|}
\hline State & $\begin{array}{c}\alpha_{R} \\
\left(\mathrm{~cm}^{3} \mathrm{~s}^{-1}\right)\end{array}$ \\
\hline \multicolumn{2}{|l|}{$T(\mathrm{~K})=1000$} \\
\hline $2 s 2 p^{3}{ }^{3} D^{o} \ldots \ldots \ldots \ldots \ldots \ldots$ & $9.17 \mathrm{E}-10$ \\
\hline $2 p^{3}{ }^{2} D^{o} 3 s^{3} D^{o}$ & $1.88 \mathrm{E}-10$ \\
\hline $2 p^{3}{ }^{2} D^{o} 4 s^{3} D^{o}$ & $6.64 \mathrm{E}-11$ \\
\hline $2 s^{2} 2 p^{2}{ }^{3} P^{e}$ & $3.38 \mathrm{E}-11$ \\
\hline $2 p^{3}{ }^{2} D^{o} 4 d^{3} F^{o}$ & $2.83 \mathrm{E}-11$ \\
\hline $2 p^{3}{ }^{2} D^{o} 4 d^{3} D^{o} \ldots \ldots \ldots \ldots$ & $2.67 \mathrm{E}-11$ \\
\hline $2 s^{2} p^{2}{ }^{2} D^{e} 5 p^{3} D^{o} \ldots \ldots \ldots \ldots$ & $2.07 \mathrm{E}-11$ \\
\hline $2 s^{2} 2 p^{2}{ }^{1} D^{e} \ldots \ldots \ldots \ldots \ldots \ldots \ldots$ & $1.78 \mathrm{E}-11$ \\
\hline 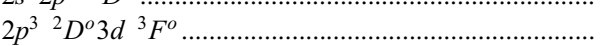 & $1.69 \mathrm{E}-11$ \\
\hline $2 s 2 p^{2}{ }^{2} S^{e} 4 f^{3} F^{o} \ldots \ldots \ldots \ldots \ldots \ldots$ & $1.61 \mathrm{E}-11$ \\
\hline $2 p^{3}{ }^{2} D^{o} 3 d^{3} D^{o} \ldots \ldots$ & $1.52 \mathrm{E}-11$ \\
\hline $2 s 2 p^{2}{ }^{2} P^{e} 3 p^{3} D^{o} \ldots \ldots \ldots \ldots$ & $8.93 \mathrm{E}-12$ \\
\hline 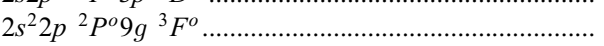 & $8.43 \mathrm{E}-12$ \\
\hline $2 s 2 p^{2}{ }^{2} S^{e} 5 g^{3} G^{e}$ & $7.80 \mathrm{E}-12$ \\
\hline $2 s^{2} 2 p^{2} P^{o} 3 d^{3} F^{o}$ & $7.70 \mathrm{E}-12$ \\
\hline $2 s^{2} 2 p^{2} P^{o} 3 p^{3} D^{e}$ & $7.12 \mathrm{E}-12$ \\
\hline $2 s 2 p^{2}{ }^{2} D^{e} 5 p^{3} F^{o}$ & $6.96 \mathrm{E}-12$ \\
\hline 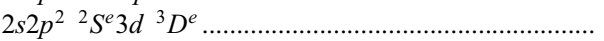 & $6.63 \mathrm{E}-12$ \\
\hline $2 s 2 p^{2}{ }^{2} D^{e} 3 p^{3} F^{o}$ & $6.31 \mathrm{E}-12$ \\
\hline $2 s^{2} 2 p^{2} P^{o} 4 p^{3} P^{e}$ & $5.61 \mathrm{E}-12$ \\
\hline 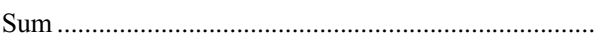 & $1.41 \mathrm{E}-09$ \\
\hline 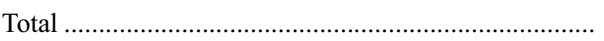 & $1.99 \mathrm{E}-09$ \\
\hline 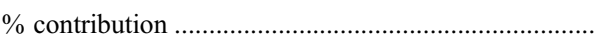 & $71 \%$ \\
\hline \multicolumn{2}{|l|}{$T(\mathrm{~K})=10,000$} \\
\hline $2 s 2 p^{3}{ }^{3} D^{o}$ & $2.76 \mathrm{E}-10$ \\
\hline $2 p^{3}{ }^{2} D^{o} 3 s^{3} D^{o}$ & $5.63 \mathrm{E}-11$ \\
\hline $2 s^{2} 2 p^{2}{ }^{3} P^{e}$ & $2.53 \mathrm{E}-11$ \\
\hline $2 p^{3}{ }^{-2} D^{o} 4 s^{3} D^{o} \ldots \ldots \ldots \ldots \ldots \ldots$ & $1.99 \mathrm{E}-11$ \\
\hline $2 s^{2} 2 p^{2}{ }^{1} D^{e} \ldots \ldots \ldots$ & $1.06 \mathrm{E}-11$ \\
\hline $2 p^{3}{ }^{2} D^{o} 4 d^{3} F^{o} \ldots$ & $8.57 \mathrm{E}-12$ \\
\hline $2 p^{3}{ }^{2} D^{o} 4 d^{3} D^{o} \ldots \ldots \ldots$ & $7.93 \mathrm{E}-12$ \\
\hline $2 s 2 p^{2}{ }^{2} D^{e} 5 p^{3} D^{o}$ & $6.21 \mathrm{E}-12$ \\
\hline $2 p^{3}{ }^{2} D^{o} 3 d^{3} F^{o}$ & $5.42 \mathrm{E}-12$ \\
\hline $2 p^{3}{ }^{2} D^{o} 3 d^{3} D^{o}$ & $4.49 \mathrm{E}-12$ \\
\hline $2 s^{2} 2 p^{2} P^{o} 4 p^{3} P^{e}$ & $4.09 \mathrm{E}-12$ \\
\hline $2 s 2 p^{2}{ }^{2} P^{e} 3 s^{1} P^{e} \ldots \ldots$. & $3.91 \mathrm{E}-12$ \\
\hline $2 s 2 p^{2}{ }^{2} P^{e} 3 p^{3} D^{o}$ & $3.06 \mathrm{E}-12$ \\
\hline $2 s 2 p^{3}{ }^{1} D^{o}$ & $2.99 \mathrm{E}-12$ \\
\hline $2 s 2 p^{3}{ }^{3} P^{o}$ & $2.80 \mathrm{E}-12$ \\
\hline $2 s 2 p^{3}{ }^{3} S^{o}$ & $2.61 \mathrm{E}-12$ \\
\hline $2 s 2 p^{2}{ }^{2} D^{e} 3 p^{3} F^{o}$ & $2.54 \mathrm{E}-12$ \\
\hline $2 s^{2} 2 p^{2} P^{o} 3 d^{3} F^{o}$ & $2.41 \mathrm{E}-12$ \\
\hline $2 s^{2} 2 p^{2} P^{o} 3 p^{3} P^{e}$ & $2.31 \mathrm{E}-12$ \\
\hline $2 s 2 p^{2}{ }^{2} D^{e} 5 p^{3} F^{o}$ & $2.29 \mathrm{E}-12$ \\
\hline Sum & $4.50 \mathrm{E}-10$ \\
\hline 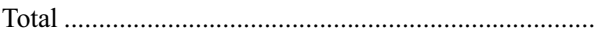 & $6.30 \mathrm{E}-10$ \\
\hline$\%$ contribution & $72 \%$ \\
\hline
\end{tabular}

\begin{tabular}{|c|c|}
\hline State & $\begin{array}{c}\alpha_{R} \\
\left(\mathrm{~cm}^{3} \mathrm{~s}^{-1}\right)\end{array}$ \\
\hline \multicolumn{2}{|l|}{$T(\mathrm{~K})=100,000$} \\
\hline $2 s^{2} 2 p^{2}{ }^{3} P^{e} \ldots \ldots \ldots$ & $1.93 \mathrm{E}-11$ \\
\hline $2 s 2 p^{3}{ }^{3} D^{o}$ & $1.46 \mathrm{E}-11$ \\
\hline $2 s^{2} 2 p^{2}{ }^{1} D^{e} \ldots \ldots \ldots \ldots$ & $1.00 \mathrm{E}-11$ \\
\hline $2 s^{2} 2 p^{2} P^{o} 4 p^{3} P^{e}$ & $4.92 \mathrm{E}-12$ \\
\hline $2 p^{3}{ }^{2} D^{o} 3 d^{3} G^{o}$ & $4.02 \mathrm{E}-12$ \\
\hline $2 s 2 p^{3}{ }^{3} S^{o} \ldots \ldots$ & $3.98 \mathrm{E}-12$ \\
\hline $2 s 2 p^{3}{ }^{3} P^{o} \ldots \ldots \ldots \ldots \ldots$ & $3.08 \mathrm{E}-12$ \\
\hline $2 s 2 p^{2}{ }^{2} D^{e} 3 d^{3} P^{e} \ldots$ & $2.55 \mathrm{E}-12$ \\
\hline $2 s^{2} 2 p^{2}{ }^{1} D^{e}$ & $2.44 \mathrm{E}-12$ \\
\hline $2 p^{3}{ }^{2} D^{o} 3 s^{3} D^{o}$ & $2.35 \mathrm{E}-12$ \\
\hline $2 p^{3}{ }^{2} D^{o} 4 d^{3} G^{o}$ & $2.27 \mathrm{E}-12$ \\
\hline $2 s^{2} 2 p^{2} P^{o} 7 i^{3} I^{o}$ & $2.23 \mathrm{E}-12$ \\
\hline $2 s 2 p^{3}{ }^{1} D^{o}$ & $2.17 \mathrm{E}-12$ \\
\hline $2 s^{2} 2 p^{2} P^{o} 3 p{ }^{3} P^{e} \ldots \ldots \ldots$ & $1.96 \mathrm{E}-12$ \\
\hline $2 s^{2} 2 p{ }^{2} P^{o} 7 i^{3} K^{o} \ldots \ldots \ldots$ & $1.92 \mathrm{E}-12$ \\
\hline $2 s^{2} 2 p^{2} P^{o} 7 i^{3} H^{o}$ & $1.87 \mathrm{E}-12$ \\
\hline $2 p^{3}{ }^{-2} D^{o} 3 d^{3} F^{o} \ldots \ldots \ldots \ldots$ & $1.49 \mathrm{E}-12$ \\
\hline $2 s 2 p^{2}{ }^{2} P^{e} 3 d^{3} F^{e} \ldots \ldots$ & $1.41 \mathrm{E}-12$ \\
\hline $2 s 2 p^{2}{ }^{2} D^{e} 3 s^{1} D^{e} \ldots \ldots \ldots$ & $1.22 \mathrm{E}-12$ \\
\hline $2 s 2 p^{3}{ }^{1} P^{o} \ldots \ldots \ldots \ldots \ldots \ldots \ldots$ & $1.14 \mathrm{E}-12$ \\
\hline Sum & $8.49 \mathrm{E}-11$ \\
\hline Total & $1.68 \mathrm{E}-10$ \\
\hline$\%$ contribution & $51 \%$ \\
\hline \multicolumn{2}{|l|}{$T(\mathrm{~K})=1,000,000$} \\
\hline $2 s^{2} 2 p^{2} P^{o} 3 d^{3} D^{o} \ldots$. & $3.87 \mathrm{E}-12$ \\
\hline $2 s^{2} 2 p^{2} P^{o} 3 d^{3} F^{o}$ & $2.11 \mathrm{E}-12$ \\
\hline $2 s^{2} 2 p^{2}{ }^{3} P^{e}$ & $1.73 \mathrm{E}-12$ \\
\hline $2 s^{2} 2 p^{2}{ }^{1} D^{e}$ & $1.51 \mathrm{E}-12$ \\
\hline $2 s^{2} 2 p^{2} P^{o} 4 f^{3} G^{e}$ & $8.49 \mathrm{E}-13$ \\
\hline $2 s 2 p^{3}{ }^{3} D^{o} \ldots \ldots \ldots$ & $7.20 \mathrm{E}-13$ \\
\hline $2 s^{2} 2 p{ }^{2} P^{o} 3 p{ }^{1} P^{e} \ldots \ldots \ldots \ldots \ldots$ & $6.78 \mathrm{E}-13$ \\
\hline $2 s^{2} 2 p^{2} P^{o} 4 p^{3} D^{e}$ & $6.43 \mathrm{E}-13$ \\
\hline $2 s^{2} 2 p^{2} P^{o} 3 p^{3} D^{e}$ & $6.25 \mathrm{E}-13$ \\
\hline $2 s 2 p^{2}{ }^{4} P^{e} 3 d^{3} D^{e}$ & $6.15 \mathrm{E}-13$ \\
\hline $2 s 2 p^{3}{ }^{3} P^{o} \ldots \ldots \ldots \ldots \ldots$ & $3.25 \mathrm{E}-13$ \\
\hline $2 s^{2} 2 p^{2} P^{o} 4 p^{3} P^{e} .$. & $3.23 \mathrm{E}-13$ \\
\hline $2 s^{2} 2 p^{2} P^{o} 3 p^{1} D^{e}$ & $2.93 \mathrm{E}-13$ \\
\hline $2 s^{2} 2 p^{2} P^{o} 4 d^{3} F^{o}$ & $2.89 \mathrm{E}-13$ \\
\hline $2 s^{2} 2 p^{2} P^{o} 4 f^{3} F^{e}$ & $2.74 \mathrm{E}-13$ \\
\hline $2 s^{2} 2 p^{2}{ }^{1} D^{e}$ & $2.72 \mathrm{E}-13$ \\
\hline $2 s 2 p^{2}{ }^{2} D^{e} 3 s^{1} D^{e}$ & $2.45 \mathrm{E}-13$ \\
\hline $2 s^{2} 2 p^{2} P^{o} 3 p^{3} P^{e}$ & $2.38 \mathrm{E}-13$ \\
\hline $2 s 2 p^{3}{ }^{3} S^{o}$ & $2.25 \mathrm{E}-13$ \\
\hline $2 p^{3}{ }^{2} D^{o} 3 d^{3} G^{o} \ldots$ & $2.02 \mathrm{E}-13$ \\
\hline Sum & $1.60 \mathrm{E}-11$ \\
\hline Total .................................. & $5.19 \mathrm{E}-11$ \\
\hline$\%$ contribution & $31 \%$ \\
\hline
\end{tabular}

states. (The complete set for a wide temperature range is available electronically.) These are of singlet and triplet symmetries that couple to the ground $2 s^{2} 2 p\left({ }^{2} P^{o}\right)$ state of the recombining target ion. A limited number of bound states usually dominate the total recombination rate at a given temperature. However, this number increases with increasing temperature as seen in Table 5. Table 5 lists the state-specific recombination rate coefficients of the first 20 dominant low- $n$ bound states of group $\mathrm{A}$, in order of their percentage contributions to the total $\alpha_{R}(T)$, at temperatures $T=1000,10,000,100,000$, and $1 \times 10^{6} \mathrm{~K}$. 
The equivalent and metastable states are usually among the dominant states because of their slow decay of background photoionization cross sections. The other states dominate depending on the positions and heights of resonances in $\sigma_{\mathrm{PI}}$, as illustrated in Figure 5. Table 5 shows that the order and the amount of contribution of individual bound states vary with temperature. The ground state may not necessarily be the dominant contributor at all temperatures, although it is one of the important ones. The present state-specific recombination rates should be close to their effective rates at these temperatures. However, above $3 \times 10^{5} \mathrm{~K}$, which corresponds to the first excited target threshold ${ }^{2} D$ for a dipole allowed transition, the state-specific rates could be underestimated somewhat since the high- $n$ DR contributions are not included individually. The level-resolved DR rates of Altun et al. (2004) consider a limited number of resonances and do not include the RR contribution.

The present results for Ar XIII should be more accurate than the available values from previous calculations since all effects are included in an ab initio manner. Based on the very good agreement between the calculated and observed energies, with the previous electron-ion recombination calculations both at very low (Aldrovandi \& Pequignot 1974) and high temperatures (Altun et al. 2004), along with accuracy of close-coupling approximation, the present rates should be accurate within $30 \%$ for the reported temperature range.

Loss of accuracy caused by exclusion of radiation damping of low- $n$ autoionizing resonances in the present work is not expected to be important. The reason is that the radiative decay rates for the dipole allowed transitions $\left(A_{f}\right.$ in Table 2) for $n=2$ states are several orders of magnitude lower than typical autoionization rate of $10^{13}-10^{14} \mathrm{~s}^{-1}$. Although radiative transition rate from the $n=3$ complex is higher, autoionization still dominates as many channels for autoionization into the lower excited states have opened up.

Inclusion of relativistic effects may improve results some. However, fine structure will increase the number of bound levels to over a thousand (Nahar 2000). In addition, increment of core fine-structure levels will require much finer energy mesh for the photoionization cross sections to resolve the narrow resonances (Zhang et al. 1999). Both Gu (2003) and Altun et al. (2004) include relativistic effects in their calculations, and their results agree with the present results very well.
Hence, the relativistic effects are not significant for the total recombination rates, although they can be exhibited in resonant features of individual states.

\section{CONCLUSIONS}

Total and partial photoionization cross sections, and total and state-specific recombination rate coefficients, are presented for Ar xIII using unified treatment for total electron-ion recombination within a close-coupling approximation and $\boldsymbol{R}$-matrix method. Self-consistency in the atomic parameters for photoionization cross sections and recombination rates is enabled by using the same wavefunction for both the inverse processes. The large-scale ab initio calculations are carried out for total photoionization cross sections of 684 bound states of Ar XIII, partial cross sections, and state-specific recombination rate coefficients for 651 bound states. In contrast to the general feature of the total recombination rate coefficient $\alpha_{R}(T)$ of smooth decay with one possible DR peak at low temperature and a typical DR bump at high temperature, $\mathrm{Ar}$ XIII shows a unique feature of multiple DR bumps in $\alpha_{\mathrm{RC}}$, two in the low- $T$ and two in high- $T$ region, not seen in any other ion.

The present results are obtained from the most detailed largescale calculations of photoionization cross sections and recombination rates for Ar XIII and should be more accurate than the existing data. The present total (RR+DR) recombination rates show very good agreement at low and high temperature with previous calculations for RR and DR rates but represent one set of rates valid for an entire range of temperatures. The overall relativistic effects are not found to be important from comparison with previous relativistic distorted wave and isolated resonance calculations.

All photoionization and recombination data are available electronically from the author.

This work has been partially supported by the US National Science Foundation and NASA. The computational work was carried out on the Cray SV1 at the Ohio Supercomputer Center. The atomic data for photoionization and recombination are available from the author at nahar.1@osu.edu and http://www. astronomy.ohio-state.edu/ nahar
Aldrovandi, S. M. V., \& Pequignot, D. 1974, Rev. Brasileira Fis., 4, 491

Altun, Z., Yumak, A., Badnell, N. R., Colgun, J., \& Pindzola, M. S. 2004, A\&A, 420, 775

Bell, R. H., \& Seaton, M. J. 1985, J. Phys. B, 18, 1589

Berrington, K. A., Burke, P. G., Butler, K., Seaton, M. J., Storey, P. J., Taylor,

K. T., \& Yan, Y. 1987, J. Phys. B, 20, 6379

Berrington, K. A., Eissner, W., \& Norrington, P. H. 1995, Comput. Phys. Commun., 92, 290

Burgess, A. 1965, ApJ, 141, 1588

Burke, P. G., Hibbert, A., \& Robb, W. D. 1971, J. Phys. B, 4, 153

Eissner, W., Jones, M., \& Nussbaumer, H. 1974, Comput. Phys. Commun., 8,270

Gu, M. F. 2003, ApJ, 590, 1131

Hummer, D. G., Berrington, K. A., Eissner, W., Pradhan, A. K., Saraph, H. E., \& Tully, J. A. 1993, A\&A, 279, 298

Nahar, S. N. 1995, ApJS, 101, 423

. 1996a, ApJS, 106, 213
REFERENCES

Nahar, S. N. 1996b, Phys. Rev. A, 53, 2417

2000, A\&AS, 147, 253

Nahar, S. N., \& Pradhan, A. K. 1992a, Phys. Rev. A, 45, 7887

1992b, Phys. Rev. Lett., 68, 1488

1994, Phys. Rev. A, 49, 1816

1995, ApJ, 447, 966

1997, ApJS, 111, 339 (Paper I)

2004, Radiation Phys. Chem., 70, 323

Pinsonneault, M. H., \& Wing, R. F. 2003, BAAS, 35, 361

Savin, D. W., et al. 2003, ApJS, 147, 421

Seaton, M. J. 1959, MNRAS, 119, 81 1987, J. Phys. B, 20, 6363

Seaton, M. J., et al. 1995-1996, the Opacity Project Team (Bristol: Inst. Phys. Publ.)

Shull, J. M., \& van Steenberg, M. 1982, ApJS, 48, 95

Yu Y., \& Seaton, M. J. 1987, J. Phys. B, 20, 6409

Zhang, H. L., Nahar, S. N., \& Pradhan, A. K. 1999, J. Phys. B, 32, 1459 